\title{
Quantum simulations and experiments on Rabi oscillations of spin qubits: Intrinsic vs extrinsic damping
}

\author{
Hans De Raedt, ${ }^{1}$ Bernard Barbara, ${ }^{2,3}$ Seiji Miyashita, ${ }^{4,5}$ Kristel Michielsen, ${ }^{6}$ Sylvain Bertaina, ${ }^{7}$ and Serge Gambarelli ${ }^{3}$ \\ ${ }^{1}$ Department of Applied Physics, Zernike Institute for Advanced Materials, University of Groningen, \\ Nijenborgh 4, NL-9747 AG Groningen, The Netherlands \\ ${ }^{2}$ Institut Néel, CNRS, Université Joseph Fourier, BP 166, FR-38042 Grenoble Cedex 9, France \\ ${ }^{3}$ Laboratoire de Chimie Inorganique et Biologique (UMR-E3 CEA-UJF), INAC, CEA-Grenoble, \\ 17 Ave. des Martyrs, FR-38054 Grenoble Cedex 9, France \\ ${ }^{4}$ Department of Physics, Graduate School of Science, The University of Tokyo, 7-3-1 Hongo, Bunkyo-Ku, Tokyo 113-8656 \\ ${ }^{5}$ CREST, JST, 4-1-8 Honcho Kawaguchi, Saitama 332-0012, Japan \\ ${ }^{6}$ Institute for Advanced Simulation, Jülich Supercomputing Centre, Research Centre Jülich, DE-52425 Jülich, Germany \\ ${ }^{7}$ IM2NP-CNRS (UMR 6242) and Université Aix-Marseille, Faculté des Sciences et Techniques, Avenue Escadrille Normandie Niemen - Case \\ 142, FR-13397 Marseille Cedex, France.
}

(Received 17 August 2011; revised manuscript received 3 November 2011; published 11 January 2012)

\begin{abstract}
Electron paramagnetic resonance experiments show that the decay of Rabi oscillations of ensembles of spin qubits depends noticeably on the microwave power, and more precisely on the Rabi frequency, an effect recently called "driven decoherence." By direct numerical solution of the time-dependent Schrödinger equation of the associated many-body system, we scrutinize the different mechanisms that may lead to this type of decoherence. Assuming the effects of dissipation to be negligible $\left(T_{1}=\infty\right)$, it is shown that a system of dipolar-coupled spins with (even weak) random inhomogeneities is sufficient to explain the salient features of the experimental observations. Some experimental examples are given to illustrate the potential of the numerical simulation approach.
\end{abstract}

DOI: 10.1103/PhysRevB.85.014408

PACS number(s): 76.30.-v, 76.20.+q, 03.65.Yz

\section{INTRODUCTION}

Decoherence generally occurs when the phase angle associated with a periodic motion is lost due to some interaction with exterior noise. In classical mechanics, it may apply to classical waves such as sound waves, seismic waves, or sea waves, whereas in quantum mechanics, it applies to the phase angles between the different components of a system in quantum superposition. The loss of phase of a quantum system may bring it to its classical regime, raising the question of whether and how the classical world may emerge from quantum mechanics. Together with the claim that decoherence is also relevant to a variety of other questions ranging from the measurement problem to the arrow of time, this underlines the important role of decoherence in the foundations of quantum mechanics. It is for all these reasons that the analysis of decoherence in quantum systems must make allowance and, in particular, must distinguish between decoherence induced by the imperfections of real systems and intrinsic decoherence induced by identified or hidden couplings to the environment. The different sources of decoherence can be classified in two main categories ${ }^{1}$ : the one-qubit decoherence coming from the coupling of individual qubits with the environment $\mathrm{t}^{2-4}$ and the multiple-qubit or pairwise decoherence coming from multiple interactions between pairs of qubits. ${ }^{5-8}$

In this paper, we take the example of paramagnetic spins because of the quality of the systems that can be elaborated (single crystals) and the possibility, offered by magnetism, to start calculations from first principles. Here, the onequbit decoherence is, in general, associated with phonons and hyperfine couplings, ${ }^{9-12}$ which are intrinsic effects, but also with nonintrinsic effects resulting from weak disorder always present in real systems of finite size: inhomogeneous fields, $g$-factor distributions, and positional distributions. Multiple-qubit decoherence is generally due to pairwise dipolar interactions with distant electronic or nuclear qubits, which is an intrinsic mechanism. ${ }^{13}$ In the following, we shall see that, more generally, when pairwise decoherence takes place in the rotating frame, extrinsic decoherence becomes crucial by itself and also by amplifying intrinsic decoherence. In particular, by way of some examples, it will be shown that the origin of driven decoherence is of the one-qubit type, with multiple possible origins (depending on the nature of the disorder). Even if dominant sources of decoherence may sometimes be identified, the complete description of decoherence and, in particular, the discrimination of intrinsic and extrinsic decoherence, is generally not accessible to experimentalists. This is a major obstacle for the reduction of decoherence, and it holds beyond magnetism. We believe that the present, pragmatic approach should be of great help in common situations where intrinsic and extrinsic decoherence mechanisms interoperate.

Assuming that each type of decoherence has its own "signature" on the Rabi oscillations, we have started a systematic study in which the Rabi oscillations of an ensemble of spins are simulated by direct numerical solution of the time-dependent Schrödinger equation (TDSE) of the associated many-body system. These simulations are performed using a parallel algorithm implementation based on a massively parallel quantum computer simulator. ${ }^{14}$ The various mechanisms that may lead to decoherence of Rabi oscillations are successively implemented in Hamiltonians, leading to different types of damping, oscillation shapes, nonzero oscillation averages, and their evolutions with exterior parameters such as the microwave power and the applied static field. The comparison with measured Rabi oscillations allows us to scrutinize the 


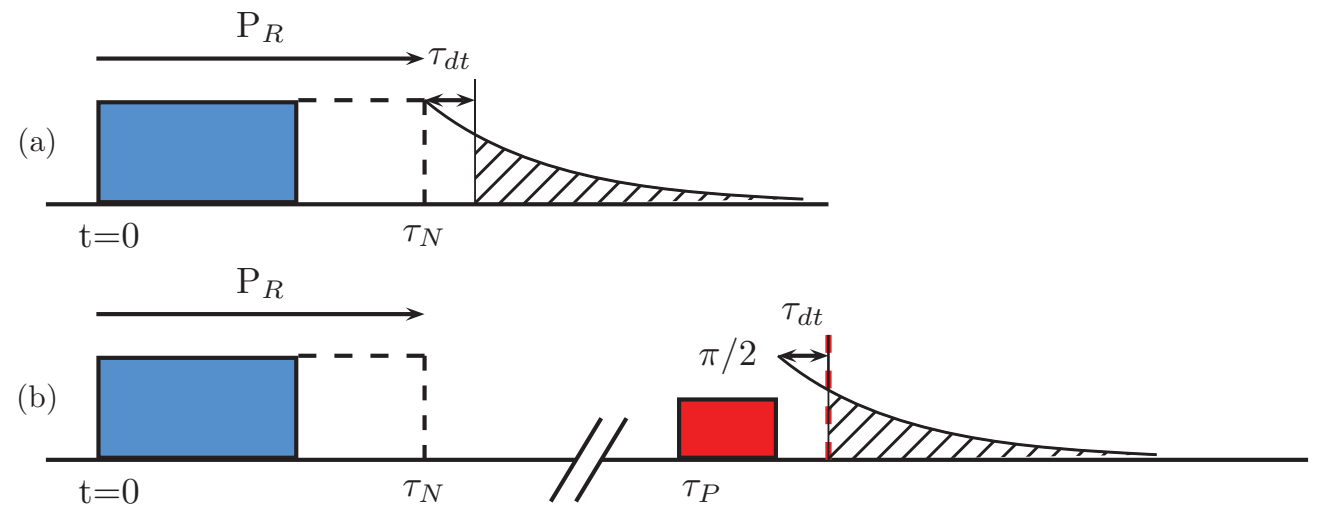

FIG. 1. (Color online) Pulse sequence used for Rabi oscillation measurements. (a) At $t=0$, a microwave pulse $P_{R}$ coherently drives the magnetization. At the end of the pulse ( $\tau=\tau_{N}$ ), the magnetization component $M^{y}(t)$ is recorded. (b) After the $P_{R}$ pulse, one waits a time much longer than $T_{2}$, but smaller than $T_{1}$, such that $M^{y}(t)$ has vanished. Then, after the waiting time $T$, a standard Hahn echo sequence of duration $\tau_{P}$ is used to measure the magnetization component $M^{z}(t)$. The dead time of the spectrometer is denoted by $\tau_{d t}$.

different decoherence mechanisms and to understand more basic aspects of decoherence, thereby opening a route to search for the optimal (intrinsic and extrinsic) ways to improve coherence of Rabi oscillations, i.e., the number of oscillations that is important for all applications.

This study is limited to the decoherence of Rabi oscillations, that is, the decoherence measured immediately after the application of a long microwave pulse. Following an earlier suggestion, ${ }^{15-17}$ it was shown that the microwave pulse inducing Rabi oscillations is itself an important source of decoherence in all the investigated systems ("driven decoherence"18-21), except when the microwave power is very small, in which case the Rabi frequency is also very small. As a consequence, the number of Rabi oscillations remains nearly constant, that is, one can not increase it by increasing the microwave power.

This observation can be quantified by comparing the damping time of Rabi oscillations (Rabi decay time $T_{R}$ ) with the usual spin-spin relaxation time $T_{2}$. The theoretical results given in this paper are all exact. Depending on the Hamiltonian parameters, the results were obtained analytically (in simple cases) and numerically (in more general cases, including dipolar interactions) and covered the large range of possibilities, namely, from $T_{R} \ll T_{2}$ up to $T_{R} \approx 2 T_{2}$ when dipolar interactions dominate (in the absence of disorder).

The systems used to compare the simulations results with experimental data are insulating single crystals of $\mathrm{CaWO}_{4}: \mathrm{Er}^{3+}, \mathrm{MgO}: \mathrm{Mn}^{2+}$, and BDPA $(\alpha-\gamma$-bisdiphenylene$\beta$-phenylally), a free radical system often used in electron paramagnetic resonance (EPR) calibration. The latter is not a diluted system, contrary to the two others, but an antiferromagnetic single crystal (identical environments) with a Néel temperature much smaller than the temperature at which our measurements are made (between 4 and $300 \mathrm{~K}$ ). These systems have been chosen in particular for the differences in their homogeneous and inhomogeneous EPR linewidths. Furthermore, in these systems, the relaxation time $T_{1}$ is much larger than $T_{2}$, as this is often the case in solid-state systems. For instance, our experiments yield a $T_{1}$ that is 10 and 40 times larger than the $T_{2}$ for $\mathrm{MgO}: \mathrm{Mn}^{2+}$ and $\mathrm{CaWO}_{4}: \mathrm{Er}^{3+}$, respectively. Therefore, as a first step in the theoretical modeling of these experiments, it is reasonable to neglect the effect of dissipation and focus on the decoherence only.

Rabi oscillations measurements have been performed in a Bruker Elexsys 680 pulse EPR spectrometer working at about $f=9.6 \mathrm{GHz}$ ( $X$ band). Depending on the sample, measurements have been done at room temperature down to liquid-helium temperature $(4 \mathrm{~K})$. The static magnetic field has always been chosen to correspond to the middle of the EPR line. The experimental procedure is illustrated in Fig. 1. A microwave pulse $P_{R}$ starts at $t=0$ and coherently drives the magnetization. At the end of the pulse $\left(\tau=\tau_{N}\right)$, the magnetization is recorded. Because of the dead time $\left(\tau_{d t}\right)$ of the spectrometer (about $80 \mathrm{~ns}$ ), it is impossible to directly measure the magnetization right after the pulse $P_{R}$. In this paper, we used two methods for the detection. The first one is simply to record the free induction decay (FID) emitted by the system when the microwave field is shut down. This method gives the value of the magnetization component $M^{y}(t)$ at the end of the pulse if we take into account two important conditions: (i) $P_{R}$ is a nonselective pulse (all spins of the line are excited) and under this condition, the FID signal is the Fourier transform of the EPR line. (ii) The EPR linewidth must be sharp enough. Since the FID is the Fourier transform of the EPR spectrum, a linewidth $\gtrsim 4 G$ will lead to a decay time of the FID less than $80 \mathrm{~ns}$, and the FID will be hidden by the dead time of the spectrometer. The second method is used when the EPR line is too broad or if one wants to probe the longitudinal magnetization $M^{z}(t)$. In this case, another probe sequence has to be used. After the $P_{R}$ pulse, one waits a time $T$ much longer than $T_{2}$ but smaller than $T_{1}$ in order that $M^{y}(t)$ vanishes. After the waiting time $T$, a standard Hahn echo sequence ( $\pi / 2-\tau-\pi-\tau-$ echo) is used to measure the longitudinal magnetization. In this paper, we do not study (a) the effects of the spin-echo pulses on the measurements and (b) the effect of temperature. For (a), this implies that the comparison with theory is through the measured so-called free-decay time $T_{2}^{*}$ (different from the usual $T_{2}$ ) in which a component of the total magnetization is directly measured through an induction coil, and for (b), that the measurements of $T_{2}^{*}$ are done at a sufficiently low temperature, which is quite easy to realize since the $T_{2}^{*}$ of $\alpha-\gamma$-bisdiphenylene- $\beta$-phenylally (BDPA) is 
nearly independent of temperature and more generally the Rabi time $T_{R}$, most important in the context of this paper, also.

The paper is organized as follows. In Sec. II, the quantum spin model is specified in detail and the simulation procedure is briefly discussed. Our results are presented in Sec. III. As there are many different cases to consider, to structure the presentation, the results have been grouped according to the kind of randomness, describing for each kind (i) the noninteracting case, (ii) the interacting case, and (iii) a comparison with experiments if this is possible. In Sec. IV, we present a model of "averaged local Bloch equations," giving a complete, exact description of one-qubit decoherence and incorporates multiple-qubit decoherence phenomenologically. A summary and outlook is given in Sec. V.

\section{MODEL}

We consider a system of $L$ dipolar-coupled spins subject to a static magnetic field along the $z$-axis and a circular polarized microwave perpendicular to the $z$-axis. The Hamiltonian reads

$$
\begin{aligned}
H= & -\mu_{B} \sum_{j=1}^{L} \mathbf{B}_{j}(t) \cdot \mathbf{g}_{j} \cdot \mathbf{S}_{j}+\frac{\mu_{0} \mu_{B}^{2}}{4 \pi} \sum_{j<k} \frac{\mathbf{S}_{j} \cdot \mathbf{g}_{j} \cdot \mathbf{g}_{k} \cdot \mathbf{S}_{k}}{\mathbf{r}_{j k}^{3}} \\
& -3 \frac{\left(\mathbf{S}_{j} \cdot \mathbf{g}_{j} \cdot \mathbf{r}_{j k}\right)\left(\mathbf{S}_{k} \cdot \mathbf{g}_{k} \cdot \mathbf{r}_{j k}\right)}{\mathbf{r}_{j k}^{5}}
\end{aligned}
$$

where $\mathbf{B}_{j}(t)=\left(B_{j}^{\prime} \cos \omega t,-B_{j}^{\prime} \sin \omega t, B_{0}\right)$ denotes the external magnetic field, composed of a large static field $B_{0}$ along the $z$-axis and a circular time-dependent microwave field $B_{j}^{\prime}$ $\left(\max _{j}\left|B_{j}^{\prime}\right| \ll \min _{j}\left|B_{0}\right|\right.$ ), which may depend on the position of the $j$ th spin, represented by the spin-1/2 operators $\mathbf{S}_{j}=$ $\left(S_{j}^{x}, S_{j}^{y}, S_{j}^{z}\right)$ with eigenvalues $\pm 1 / 2$. The vector $\mathbf{r}_{j k}$ connects the positions of spins $j$ and $k$. It is assumed that the $g$ tensor can be written as $\mathbf{g}_{j}=g_{e}\left(\mathbb{1}+\Delta \mathbf{g}_{j}\right)$, where the perturbation $\Delta \mathbf{g}_{j}$ is a random matrix.

As usual in the theory of NMR/ESR, we separate the fast rotational motion induced by the large static field $B_{0}$ from the remaining slow motion by a transformation to the reference frame that rotates with an angular frequency determined by $B_{0}$. Taking the ideal, noninteracting system without fluctuations in the $g$ tensors as the reference system, we define $\omega_{0}=g_{e} \mu_{B} B_{0}$ and assume from now on that this ideal system is at resonance, that is, the microwave frequency is given by $\omega=\omega_{0}$.

The transformation to the reference frame rotating with angular frequency $\omega_{0}$ is defined by

$$
X_{R F}=\exp \left(i t \omega_{0} \sum_{j=1}^{L} S_{j}^{z}\right) X \exp \left(-i t \omega_{0} \sum_{j=1}^{L} S_{j}^{z}\right)
$$

where $X$ denotes any combination of spin operators. Transforming Eq. (1) to the rotating frame, we find that $H_{R F}$ contains contributions that (i) do not depend on time, (ii) have factors $e^{i t \omega_{0}}$ or $e^{-i t \omega_{0}}$, or (iii) have factors $e^{2 i t \omega_{0}}$ or $e^{-2 i t \omega_{0}}$. Contributions that depend on time oscillate very fast (because $\omega_{0}$ is large) and, according to standard NMR/ESR theory, may be neglected, which we have confirmed for a few cases. The remaining time-independent, secular terms yield the Hamiltonian

$$
\begin{aligned}
H_{R F}= & -\omega_{0} \sum_{j=1}^{L} \frac{g_{j}^{z}-g_{e}}{g_{e}} S_{j}^{z}-\mu_{B} g_{e} \sum_{j=1}^{L} B_{j}^{\prime} \frac{g_{j}^{x}+g_{j}^{y}}{2 g_{e}} S_{j}^{x} \\
& +\frac{\mu_{0} \mu_{B}^{2} g_{e}^{2}}{4 \pi} \sum_{j<k} \frac{g_{j}^{z} g_{k}^{z}\left[1-3 z_{j k}^{2} / r_{j k}^{2}\right]}{g_{e}^{2} r_{j k}^{3}} S_{j}^{z} S_{k}^{z} \\
& +\frac{g_{j}^{x} g_{k}^{x}\left[1-3 x_{j k}^{2} / r_{j k}^{2}\right]+g_{j}^{y} g_{k}^{y}\left[1-3 y_{j k}^{2} / r_{j k}^{2}\right]}{2 g_{e}^{2} r_{j k}^{3}} \\
& \times\left(S_{j}^{x} S_{k}^{x}+S_{j}^{y} S_{k}^{y}\right) .
\end{aligned}
$$

From Eq. (3), it is clear that variations in $g_{j}^{z}$ have the same effect as local variations (inhomogeneities) in the static magnetic field. Inhomogeneities in the microwave field and the variations in $\left(g_{j}^{x}, g_{j}^{y}\right)$ are cumulative. Although the variations in $g_{j}^{x}, g_{j}^{y}$, and $g_{j}^{z}$ also affect the dipolar interactions, these effects may be difficult to distinguish from the effect of the positional disorder of the spins in the solid, in particular if the spins are distributed randomly. Note that the total magnetization $M^{z}=\sum_{j=1}^{L} S_{j}^{z}$ commutes with the dipolar terms in Eq. (3). Therefore, neglecting the terms that oscillate with $\omega_{0}$ or $2 \omega_{0}$, the longitudinal magnetization $M^{z}(t)=\sum_{j=1}^{L} S_{j}^{z}$ is a constant of motion in the absence of a microwave field $\left(B_{j}^{\prime}=0\right)$.

If all the $g$ 's are the same and equal to $g_{e}$, the Hamiltonian (3) reduces to the familiar expression

$$
\begin{aligned}
H_{R F}= & -\sum_{j=1}^{L} h_{j} S_{j}^{x}+\frac{\mu_{0} \mu_{B}^{2} g_{e}^{2}}{4 \pi} \sum_{j<k} \frac{\left[1-3 z_{j k}^{2} / r_{j k}^{2}\right]}{r_{j k}^{3}} \\
& \times\left[S_{j}^{z} S_{k}^{z}-\frac{1}{2}\left(S_{j}^{x} S_{k}^{x}+S_{j}^{y} S_{k}^{y}\right)\right]
\end{aligned}
$$

of the Hamiltonian of dipolar-coupled spins in the reference frame that rotates at the resonance frequency $\omega_{0}$.

\section{A. Simulation model}

We now specify the model as it will be used in our computer simulations. We rewrite the Hamiltonian (3) as

$$
\begin{aligned}
H_{R F} / \hbar= & -2 \pi F_{0} \sum_{j=1}^{L} \xi_{j}^{z} S_{j}^{z}-2 \pi h_{p} F_{R} \sum_{j=1}^{L} \frac{\left(1+\zeta_{j}\right)\left(2+\xi_{j}^{x}+\xi_{j}^{y}\right)}{2} S_{j}^{x}+2 \pi D_{0} \sum_{j<k} \frac{\left(1+\xi_{j}^{z}\right)\left(1+\xi_{k}^{z}\right)\left[1-3 z_{j k}^{2} / r_{j k}^{2}\right]}{r_{j k}^{3}} S_{j}^{z} S_{k}^{z} \\
& +2 \pi D_{0} \sum_{j<k} \frac{\left(1+\xi_{j}^{x}\right)\left(1+\xi_{k}^{x}\right)\left[1-3 x_{j k}^{2} / r_{j k}^{2}\right]+\left(1+\xi_{j}^{y}\right)\left(1+\xi_{k}^{y}\right)\left[1-3 y_{j k}^{2} / r_{j k}^{2}\right]}{2 r_{j k}^{3}}\left(S_{j}^{x} S_{k}^{x}+S_{j}^{y} S_{k}^{y}\right),
\end{aligned}
$$


where we take $F_{0}=\omega_{0} / 2 \pi \hbar=9.7 \mathrm{GHz}$ for the Larmor frequency induced by the large static field, $F_{R}=55.96 \mathrm{MHz}$ denotes the Rabi frequency for an isolated spin in a microwave field of $1 \mathrm{mT}$, we introduce $h_{p}$ as a parameter to control the amplitude of the microwave pulse, $D_{0}=51.88 \mathrm{GHz}$, and we express all distances in $\AA$. With this choice of units, it is convenient to express frequencies in $\mathrm{MHz}$ and time in $\mu \mathrm{s}$. The new dimensionless variables $\xi_{j}^{\alpha}$ for $\alpha=x, y, z$ and $\zeta_{j}$ are defined by $g_{j}^{\alpha}=g_{e}\left(1+\xi_{j}^{\alpha}\right)$ and $\mu_{B} g_{e} B_{j}^{\prime} / \hbar=$ $2 \pi h_{p} F_{R}\left(1+\zeta_{j}\right)$, respectively. For concreteness, we assume that the spins are located on the Si diamond lattice with lattice parameter $5.43 \AA$ (to a first approximation, the choice of the lattice is not expected to affect the results). Not every lattice site is occupied by a spin: We denote the concentration of spins (number of spins $/ \AA^{3}$ ) by $n$. In experiment, $n \approx 10^{-4}, \ldots$, $10^{-6}$.

Guided by experimental results, we assume that the distribution of $\xi_{j}^{\alpha}$ is Lorenztian and independent of $\alpha$, cut off at $\xi_{0}$, and has a width $\Gamma$ :

$$
p\left(\xi_{j}^{\alpha}\right)=\frac{1}{\arctan \left(\xi_{0} / \Gamma\right)} \frac{\Gamma}{\left(\xi_{j}^{\alpha}\right)^{2}+\Gamma^{2}} \Theta\left(\xi_{0}-\left|\xi_{j}^{\alpha}\right|\right) .
$$

The reason for introducing the cutoff $\xi_{0}>0$ is that because the Lorentzian distribution has a very long tail, in practice, we may generate $\xi^{\prime} s$ such that the corresponding value of $g$ is negative, which may not be physical. Therefore, we use

$$
\xi_{j}^{\alpha}=\Gamma \tan \left[(2 r-1) \arctan \left(\xi_{0} / \Gamma\right)\right],
$$

to generate the random variables $\xi_{j}^{\alpha}$ with distribution Eq. (6) from uniformly distributed random numbers $0<r<1$. Likewise, the microwave amplitudes are given by $B_{j}^{\prime}=h_{p}\left(1+\zeta_{j}\right)$, where the $\zeta$ 's are random numbers with distribution

$$
p\left(\zeta_{j}\right)=\frac{1}{\arctan \left(\zeta_{0} / \gamma\right)} \frac{\gamma}{\zeta_{j}^{2}+\gamma^{2}} \Theta\left(\zeta_{0}-\left|\zeta_{j}\right|\right),
$$

and $h_{p}$ is the average amplitude of the microwave field. Appendix A gives a summary of the model parameters that we use in our simulations.

\section{B. Simulation procedure}

The physical properties of interest, in particular, the decay rate $c_{\mathrm{R}}=1 / T_{R}$ of the Rabi oscillations and the intrinsic decay rate $c_{2}=1 / T_{2}$, can be extracted from the time dependence of the longitudinal and transverse magnetization, respectively, and are defined by

$$
\begin{aligned}
\left\langle M^{z}(t)\right\rangle & =\sum_{j=1}^{L}\left\langle S_{j}^{z}\right\rangle=\sum_{j=1}^{L}\left\langle\Psi(t)\left|S_{j}^{z}\right| \Psi(t)\right\rangle, \\
\left\langle M^{x}(t)\right\rangle=\sum_{j=1}^{L}\left\langle S_{j}^{x}\right\rangle & =\sum_{j=1}^{L}\left\langle\Psi(t)\left|S_{j}^{x}\right| \Psi(t)\right\rangle,
\end{aligned}
$$

respectively. We compute the time-dependent wave function $|\Psi(t)\rangle$ by solving the TDSE

$$
i \hbar \frac{\partial}{\partial t}|\Psi(t)\rangle=H_{R F}|\Psi(t)\rangle,
$$

with $H_{R F}$ given by Eq. (5). Numerically, we solve the TDSE using an unconditionally stable product-formula algorithm. ${ }^{22}$ For the largest spin systems, we perform the simulations using a parallel implementation of this algorithm, based on a massively parallel quantum computer simulator. ${ }^{14}$ Our numerical method strictly conserves the norm of the wave function and conserves the energy to any desired precision (limited by the machine precision).

In analogy with the experimental procedure, we carry out two types of simulations yielding the longitudinal (transverse) magnetization $\left\langle M^{z}(t)\right\rangle\left[\left\langle M^{x}(t)\right\rangle\right]$. From Eq. (5), it follows directly that $d\left\langle M^{z}(t)\right\rangle / d t=-\left\langle M^{y}(t)\right\rangle$, hence, $\left\langle M^{z}(t)\right\rangle$ is directly related to $\left\langle M^{y}(t)\right\rangle$ measured in experiment. We prepare the spin system, that is, the state $|\Psi(0)\rangle$, such that all spins are aligned along the $z(x)$-axis. Then, for a fixed value of the microwave amplitude $h_{p}\left(h_{p}=0\right)$ and a particular realization of the random variables $\xi_{j}^{x}, \xi_{j}^{y}, \xi_{j}^{z}, \zeta_{j}$ and the distribution of the spins on the lattice, we solve the TDSE and compute Eqs. (9) and (10). This procedure is then repeated several times with different realizations of random variables and distributions of spins. Finally, we compute the average of Eqs. (9) and (10) over all these realizations and analyze its time dependence by fitting a simple, damped sinusoidal function to the simulation data. This then yields the decay rate $c_{\mathrm{R}}=1 / T_{R}$ (intrinsic decay rate $c_{2}=1 / T_{2}$ ) of the Rabi oscillations.

\section{RESULTS}

In the sections that follow, we consider the various sources of decoherence separately. We also study the interplay of intrinsic decoherence due to, e.g., pairwise interactions and extrinsic decoherence due to, e.g., single spins driven by external magnetic fields when different spins have different environments (different couplings to static and microwave fields). The averaging over different spins leads to decoherence, that is, phase destruction of the electromagnetic waves generated by the spins. These two types of decoherence lead to the observed damping of Rabi oscillations, which takes place through energy exchange between the spin system and the applied microwave field. In the following, we show that energy dissipation from the spin bath to the electromagnetic bath is sufficient to explain the experimental results on the Rabi decay time. This is the reason why we neglect, in this paper, the dissipation effect of phonons, (our spin-lattice relaxation time $T_{1}$ is infinite). Note that if we turn off the microwave field, the longitudinal component of the magnetization commutes with the Hamiltonian (5) and hence does not change with time at all, showing that energy exchange with the electromagnetic bath is essential. In the following sections, we give two examples in which energy flows from the electromagnetic bath to the spin bath and from the spin bath to the electromagnetic bath.

\section{A. Fixed $g$-factors and homogeneous fields}

In the absence of randomness on the $g$-factors or on the microwave amplitude, the Hamiltonian is given by Eq. (5) with $\xi^{x}=\xi^{y}=\xi^{z}=\zeta=0$. 

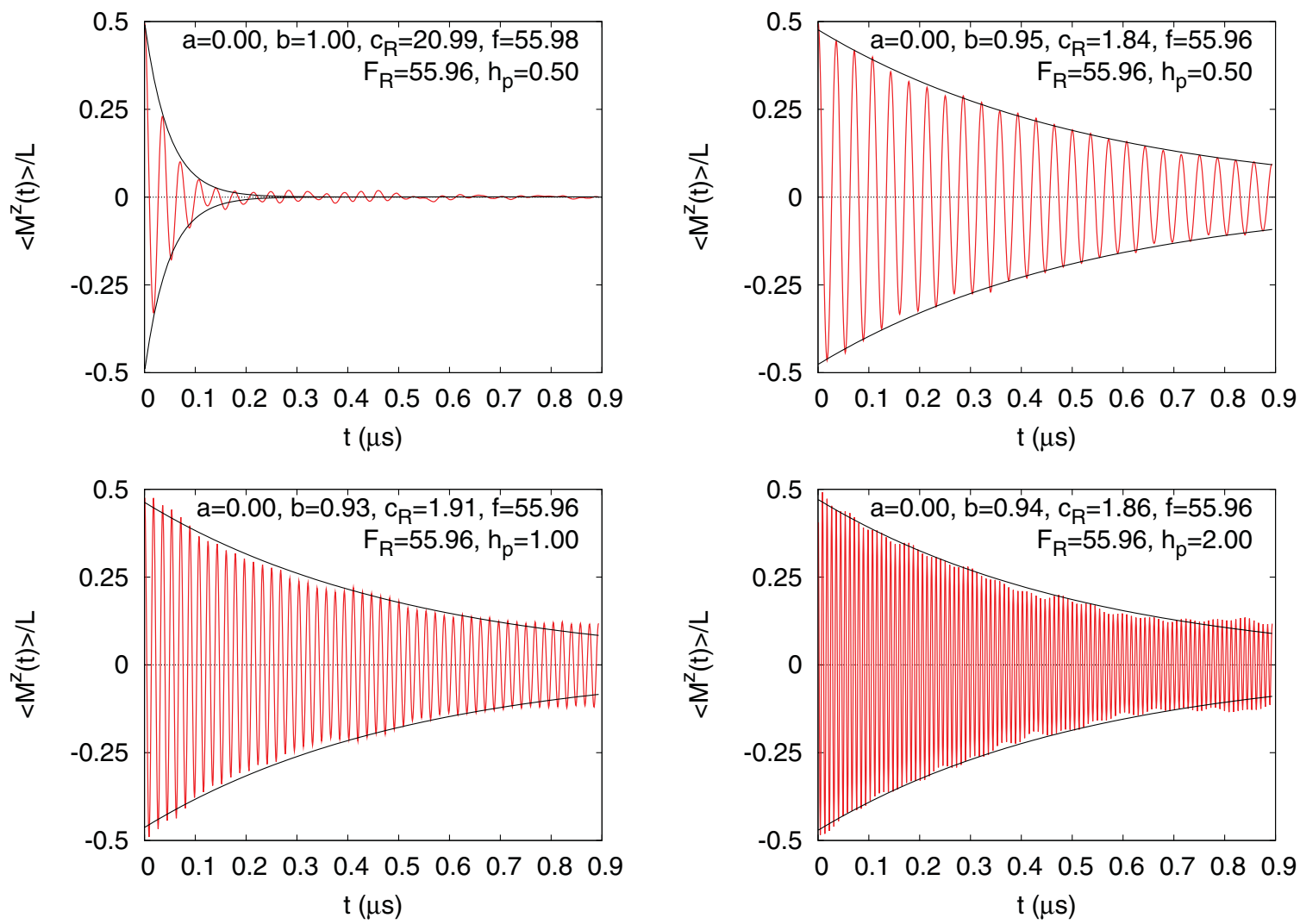

FIG. 2. (Color online) The Rabi oscillations of the longitudinal magnetization as obtained by solving the TDSE for the Hamiltonian (5) for 26 dipolar-coupled spins for two different concentrations $n$, without random fluctuations in the $g$-factors $(\Gamma=0)$ and on the microwave amplitude $(\gamma=0)$. Top left: $n=10^{-3}$. Top right and bottom: $n=10^{-4}$. The solid lines represent the envelope $\left(a \pm b e^{-c_{\mathrm{R}} t}\right) / 2$ of the function $\left(a+b e^{-c_{\mathrm{R}} t} \cos 2 \pi f t\right) / 2$ that was fitted to the data.

\section{Noninteracting spins}

For noninteracting spins, we can drop the spin label and write the Hamiltonian (in the rotating frame) for a single spin as

$$
H_{R F} / \hbar=-2 \pi h_{p} F_{R} S^{x} .
$$

The time evolution of the longitudinal magnetization takes the simple form

$$
\left\langle\Psi(t)\left|S^{z}\right| \Psi(t)\right\rangle=\frac{1}{2} \cos \Omega_{R} t,
$$

showing that the $z$ component of the spin performs undamped Rabi oscillations with angular frequency $\Omega_{R}=2 \pi h_{p} F_{R}$. Therefore, $T_{R}=\infty$. Furthermore, the transverse magnetization is conserved and, therefore, $T_{2}=\infty$. Summarizing, in the absence of randomness and dipole-dipole interactions, we have

$$
T_{R}=\infty, \quad T_{2}=\infty .
$$

\section{Dipolar-coupled spins}

In Figs. 2 and 3, we present simulation results for the longitudinal and transverse magnetization, respectively, as obtained by averaging the solutions of the TDSE over 10 different distributions of 26 dipolar-coupled spins on the lattice. Our simulation results, many of them not shown, lead us to the following conclusions: (i) For both concentrations $n=10^{-3}$ and $10^{-4}$ and for microwave amplitudes $h_{p}=0.5,1,2$, the Rabi oscillations decay exponentially. Indeed, the fits are good, as indicated by the small differences between the Rabi frequency $\left(F_{R}=\right.$ 55.96) and the values of $f$ obtained by the fitting procedure.

(ii) The decay rate $c_{\mathrm{R}}=1 / T_{R}$ increases with $n$, with a slope of approximately 1.7 (data not shown).

(iii) Within the statistical fluctuations resulting from the random distribution of the spins on the lattice, $c_{\mathrm{R}}=1 / T_{R}$ does not depend on the microwave amplitude $h_{p}$, but strongly depends on the concentration $n$.

(iv) Simulations (data not shown) for $n=0.25 \times$ $10^{-4}, \ldots, 10^{-3}$ indicate that $T_{2} \propto n$, as expected theoretically.

(v) The simulation data suggest that $c_{2}=1 / T_{2}>c_{\mathrm{R}}=$ $1 / T_{R}$

Summarizing, in the absence of local randomness but in the presence of dipole-dipole interactions, we have

$$
T_{R}=T_{R}(n)>T_{2}=T_{2}(n) .
$$

\section{Experimental results: BDPA}

We now compare these theoretical predictions to experiments performed on a single crystal of BDPA $(\alpha-\gamma-$ bisdiphenylene- $\beta$-phenylally). With a linewidth of $0.09 \mathrm{mT}$, this system is quite homogeneous with a very narrow distribution of the $g$-factors. Moreover, the sample used was very tiny such that we may consider the microwave to be 

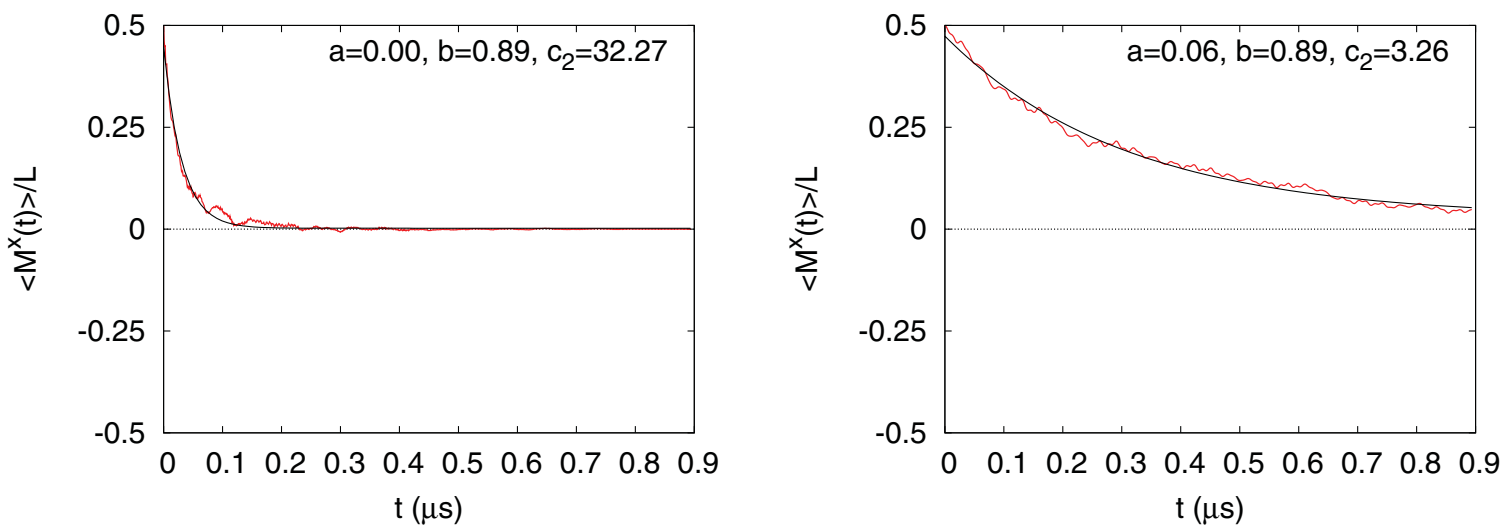

FIG. 3. (Color online) Time evolution of the transverse magnetization as obtained by solving the TDSE for the Hamiltonian (5) for 26 dipolar-coupled spins without random fluctuations in the $g$-factors $(\Gamma=0)$ and on the microwave amplitude $(\gamma=0)$. Left: $n=10^{-3}$. Right: $n=10^{-4}$. The nonzero value of $a$ is due to the statistical noise and the relatively short-time interval used for the fit. The solid line represents the function $\left(a+b e^{-c_{2} t}\right) / 2$ that was fitted to the data.

homogeneous inside the sample. Results are presented in Fig. 4. They show an example of Rabi oscillations obtained from FID measurements. The Rabi oscillations fit very well to

$$
M^{y}(t)=A_{0} \sin \left(\Omega_{R} t+\phi\right) \exp \left(-t / T_{R}\right)+M^{y}(\infty),
$$

for all microwave powers. The obtained Rabi decay time $T_{R}$ is clearly independent of the amplitude of the microwave field, as predicted by the model when $\Gamma=\gamma=0$. It is also very close to $T_{2}^{*}$, the FID decay time given by the Fourier transform of the EPR linewidth. This is also in agreement with predictions when $\Gamma=\gamma=0$ and $D_{0} \neq 0, T_{2}^{*}$ being a coherence time fully

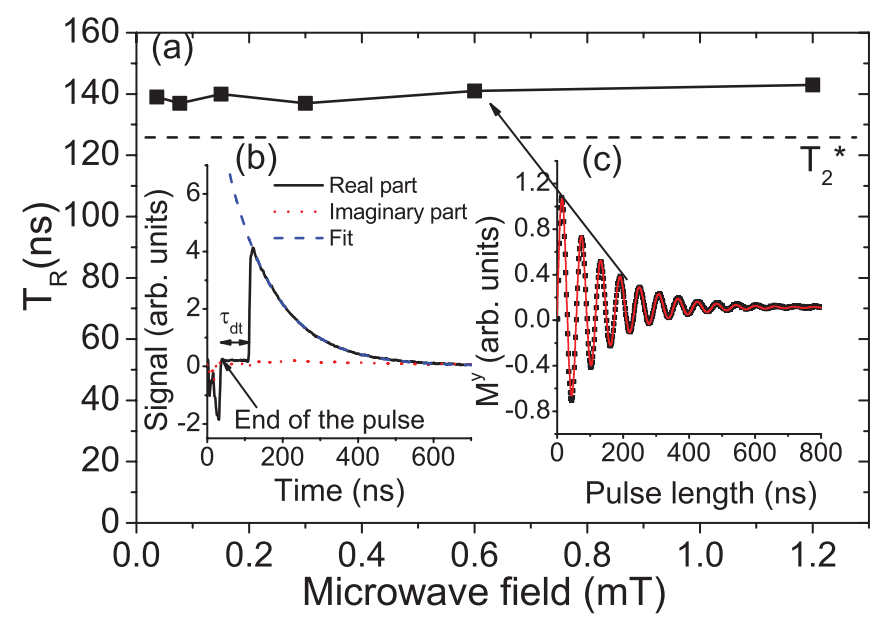

FIG. 4. (Color online) (a) Decay time $T_{R}$ of the Rabi oscillation of BDPA as a function of the amplitude of the microwave field. Dashed line: Value of $T_{2}^{*}$, the decay time of the FID. (b) Example of the FID signal. $\tau_{d t}$ : Dead time of the spectrometer. Solid line (back): In-phase (real part of the) FID signal recorded by the detector. Dashed line (blue): Best fit to the exponential decay. Dotted line (red): Out-of-phase (imaginary part of the) signal, minimized by adjusting the phase. (c) Example of Rabi oscillations. The red solid line is the best fit to $M^{y}(t)=A_{0} \sin \left(\Omega_{R} t+\phi\right) \exp \left(-t / T_{R}\right)+M^{y}(\infty)$, giving the Rabi decay time $T_{R}$ and a non-zero offset. The nonzero value of $M^{y}(\infty)$ is due to dissipation effects, ${ }^{23}$ collectively described by the relaxation time $T_{1}$, which are not included in the microscopic model considered in the present paper. Measurements were carried out at room temperature. equivalent to $T_{2}$. The discrepancy between $T_{R}(\sim 140 \mathrm{~ns})$ and $T_{2}^{*}(=128 \mathrm{~ns})$ is due to a small inhomogeneous broadening (about 10\%).

\section{B. Randomness in the microwave amplitude only}

In the case of randomness in the microwave amplitude only, the Hamiltonian is given by Eq. (5) with $\xi^{x}=\xi^{y}=\xi^{z}=0$. Such a randomness is inherent to finite-size cavities and becomes smaller as the size of the sample relative to the size of the cavity is reduced.

\section{Noninteracting spins}

For noninteracting spins $\left(D_{0}=0\right)$, we can readily compute the average over the distribution of $\zeta_{j}$ analytically if we neglect the cutoff of the Lorentzian distribution. As all spins are equivalent, we may drop the spin index $j$ and we obtain

$$
\overline{\left\langle S^{z}(t)\right\rangle}=\frac{\gamma}{2 \pi} \int_{-\infty}^{+\infty} \frac{\cos \Omega_{R}(1+\zeta) t}{\zeta^{2}+\gamma^{2}} d \zeta=\frac{1}{2} e^{-\gamma \Omega_{R} t} \cos \Omega_{R} t,
$$

showing that the Rabi oscillations decay exponentially and that the decay time of the Rabi oscillations is given by $1 / T_{R}=$ $\gamma \Omega_{R}$. Furthermore, the transverse magnetization is conserved and therefore $T_{2}=\infty$. Summarizing, in the presence of randomness in the microwave field only and in the absence of dipole-dipole interactions, we have

$$
1 / T_{R}=\gamma \Omega_{R}>1 / T_{2}=0,
$$

showing that the decay rate of the Rabi oscillations increases linearly with the microwave amplitude $h_{p}$, whereas $T_{2}$ remains infinite. This is easy to understand: $T_{2}$ is infinite due to the lack of pairwise intrinsic decoherence, whereas destructive interference associated with weak positional randomness in $h_{p}$ (the microwave field) leads to a reduction of $T_{R}$ when $h_{p}$ increases (one-qubit decoherence).

\section{Interacting spins: Dipole-dipole interaction}

In Fig. 5, we present simulation results for systems of 12 spins with dipole-dipole interaction and randomness in $h_{p}$, as obtained by averaging over 100 different realizations, 

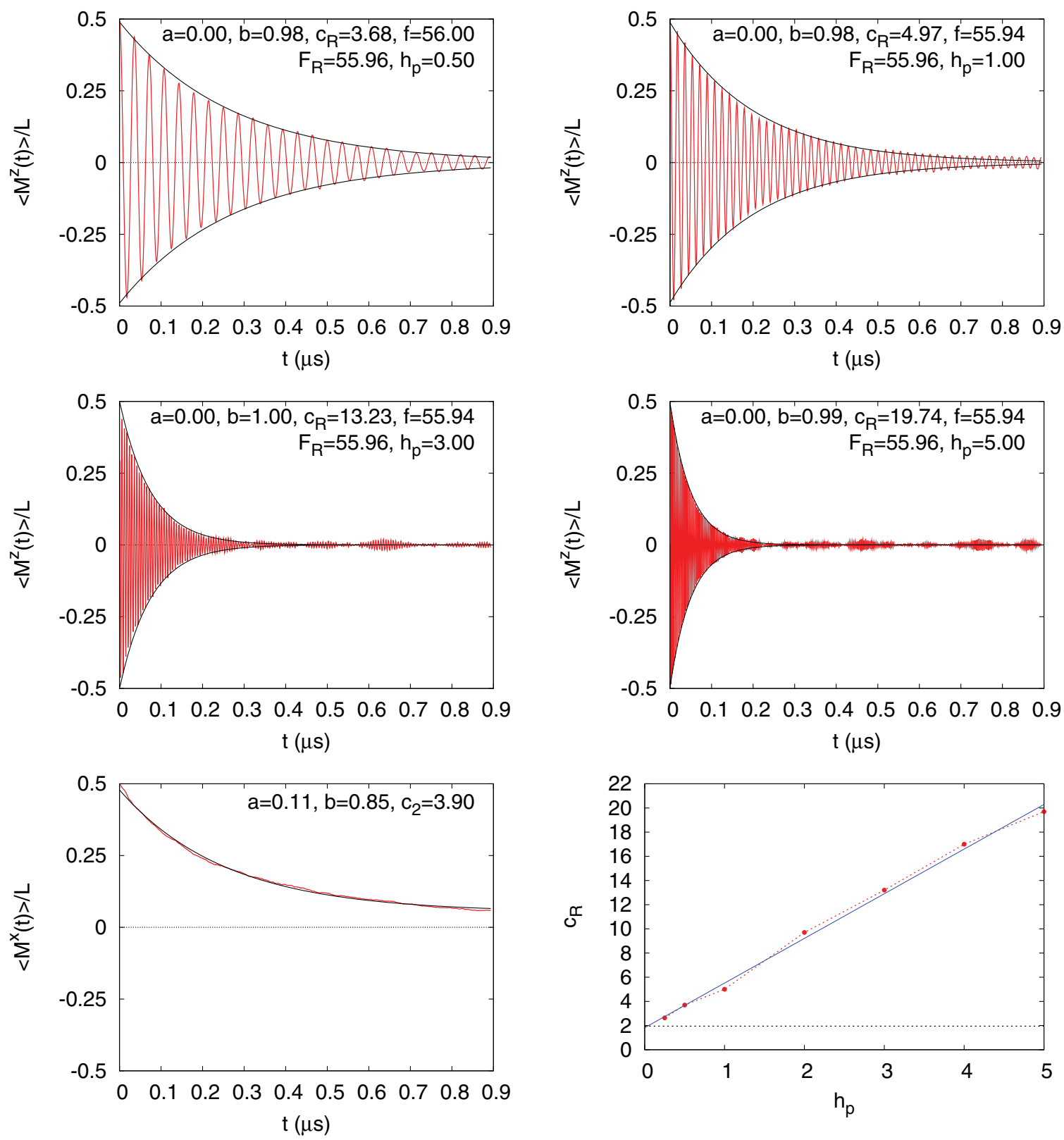

FIG. 5. (Color online) Simulation results as obtained by solving the TDSE for the Hamiltonian (5) for 12 spins (concentration $n=10^{-4}$ ) that interact via dipole-dipole interaction, without random fluctuations in the $g$-factors $(\Gamma=0)$ but with random fluctuations on the microwave amplitude $(\gamma=0.01)$. The results represent the average of 100 different realizations of 12 -spin systems. Top left to middle right: Longitudinal magnetization for different values of $h_{p}$. The solid line represents the envelope $\left(a \pm b e^{-c_{\mathrm{R}} t}\right) / 2$ of the function $\left(a+b e^{-c_{\mathrm{R}} t} \cos 2 \pi f t\right) / 2$ that was fitted to the data. Bottom left: Transverse magnetization in the absence of the microwave field $\left(h_{p}=0\right)$. The solid line represents the function $\left(a+b e^{-c_{2} t}\right) / 2$ that was fitted to the data. Bottom right: Bullets show the inverse relaxation time $c_{\mathrm{R}}=1 / T_{R}$ as a function of the microwave amplitude $h_{p}$. The dashed line connecting the bullets is a guide to the eye only. A linear fit to the simulation data yields $c_{\mathrm{R}}=1 / T_{R} \approx 3.69 h_{p}+1.82$ and is shown by the solid line. The horizontal line represents the value of $c_{2}=1 / 2 T_{2} \approx 1.95$, estimated from the data of the transverse magnetization (see bottom left).

meaning 100 different distributions of the 12 spins on the lattice. The four upper panels of Fig. 5 show results for the longitudinal magnetization $\left\langle M^{z}(t)\right\rangle$.

Rabi oscillations are damped but have zero offset. The inverse Rabi time $1 / T_{R}=c_{\mathrm{R}}$, deduced from sinusoidal fits, increases linearly with the microwave field, that is, with the Rabi frequency $\Omega_{R}$ (bottom right). Its value at $h_{p}=0$ is to good accuracy equal to $1 / 2 T_{2}\left(a_{0} \approx 1.82\right.$ for $\left.n=10^{-4}\right)$.
The slope $a_{1} \approx 3.69 / F_{R}$ is related to the matrix of the gyromagnetic factor and to the root mean square of local fields resulting from the randomness in the microwave field. These results, specific to a $h_{p}$ distribution, agree qualitatively with recently published results of the damping time of Rabi oscillations in the limit of a large inhomogeneous linewidth. ${ }^{24}$

The results for the transverse magnetization $\left\langle M^{x}(t)\right\rangle$ in the absence of microwaves $\left(h_{p}=0\right)$ are presented in the bottom 
left panel of Fig. 5. It clearly decays exponentially, as this is the case with the longitudinal magnetization. Summarizing, from Fig. 5 we conclude that, in the presence of randomness in the microwave field and of dipole-dipole interactions, we have

$$
c_{\mathrm{R}}=1 / T_{R} \approx a_{1} \Omega_{R}+a_{0}, \quad a_{0} \approx 1 / 2 T_{2} .
$$

Here, pairwise decoherence affects $T_{2}$, which is now finite (and proportional to $1 / n$ as in the case without randomness, see Sec. III A) and randomness in microwave amplitude $h_{p}$ affects $T_{R}$, which is essentially proportional to $1 / h_{p}$ at large $h_{p}$. As $T_{R}<T_{2}$, we can say that, in this case, energy flows from the spin bath to the electromagnetic bath, leading to energy dissipation in the spin bath.

\section{Experimental results: $\mathrm{CaWO}_{4}: \mathrm{Er}^{3+}$ and $\mathrm{MgO}: \mathrm{Mn}^{2+}$}

In order to show the effect of concentration on Rabi damping, we measure two samples of $\mathrm{CaWO}_{4}: \mathrm{Er}^{3+}$ with erbium concentration $0.01 \%$ and $0.001 \%$, respectively. The two samples have nearly the same shape, keeping the inhomogeneity of microwave field constant. To remove the effects of zero microwave field decay (that is, $T_{2}$ due to multispin or pairwise decoherence), we plot $1 / T_{R}-1 / T_{R 0}$, where $T_{R 0} \approx$ $1 / 2 T_{2}$ is the decay time at zero microwave field. The results are presented in Fig. 6 . The inverse Rabi decay time fits very well to $1 / T_{R}=1 / T_{R 0}+\beta \Omega_{R} / 2 \pi$, where $\beta$ is a fitting parameter. From Fig. 6, it is clear that the Rabi decay time $T_{R}$ decreases with the concentration $n$, in concert with the simulation results.

Evidence of the effect of microwave field inhomogeneity on the Rabi oscillation decay has been recently given for a sample of $\mathrm{Cr}: \mathrm{CaWO}_{4}{ }^{25}$ To provide further evidence, we took a sample of $\mathrm{MgO}$ doped with about $0.001 \%$ with $\mathrm{Mn}^{2+}$ and cut the sample into a large $\left(3.6 \times 5 \times 0.5 \mathrm{~mm}^{3}\right)$ and small $\left(1 \times 1 \times 0.5 \mathrm{~mm}^{3}\right)$ piece. At this extremely low concentration, the dipole-dipole interaction effect on the Rabi decay is negligible, hence, disorder essentially due to the microwave field inhomogeneity inside these samples will be different. Figure 7 shows the Rabi oscillations for these two samples. All parameters (microwave power, temperature, crystal orientation) are the same for the measurements on these two

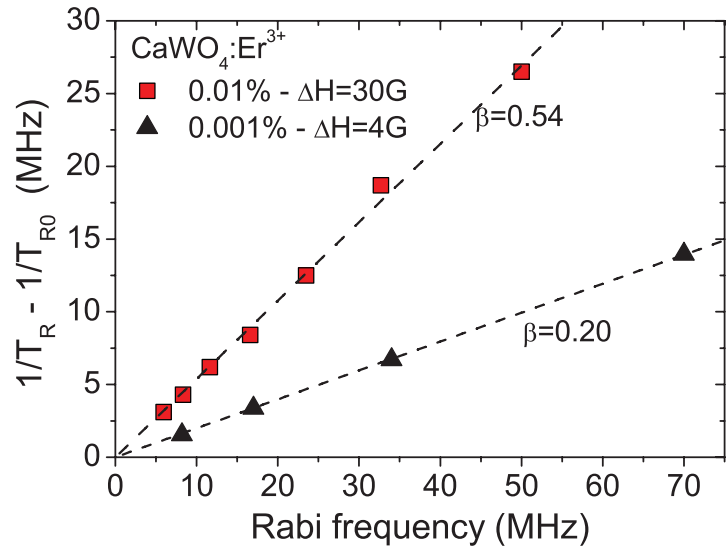

FIG. 6. (Color online) Decay time $T_{R}$ of Rabi oscillations in $\mathrm{CaWO}_{4}$ as a function of the microwave field amplitude (corresponding to the Rabi frequency $\Omega_{R}$ ) for two concentrations of the $\mathrm{Er}^{3+}$ spins. The static field $H_{0}$ is parallel to the $c$ axes of the crystal and the temperature $T \sim 4 \mathrm{~K}$. Dashed lines: Fit to $1 / T_{R}=1 / T_{R 0}+\beta \Omega_{R} / 2 \pi$. Measurements were carried out at $4 \mathrm{~K}$.

samples. The effect of the inhomogeneity of the microwave field on the Rabi decay time is clearly seen as the damping in the large sample (red line) is almost two times larger than the one in the small sample (black line).

\section{Randomness in the $g$-factors only}

We assume that there are no random fluctuations in the amplitude of the microwave pulse and that the $g$-factors fluctuate randomly from spin to spin. This effect is generally due to weak crystal distortions, imperfections, leading to small variations of crystal-field parameters.

\section{Randomness in $g^{z}$ : Noninteracting spins}

In this case, the Hamiltonian is given by Eq. (5) with $\zeta_{j}=$ $\xi^{x}=\xi^{y}=D_{0}=0$. As we then have a system of independent spins, we may drop the spin index $j$. In the case wherein, initially, all the spins are aligned along the $z$-axis, we find

$$
\begin{aligned}
\overline{\left\langle S^{x}(t)\right\rangle}= & 0, \\
\overline{\left\langle S^{y}(t)\right\rangle}= & -\frac{1}{2} \int_{-\infty}^{+\infty} \frac{h_{p} F_{R} \sin \left[2 \pi t \sqrt{\left(F_{0} \xi^{z}\right)^{2}+\left(h_{p} F_{R}\right)^{2}}\right]}{\sqrt{\left(F_{0} \xi^{z}\right)^{2}+\left(h_{p} F_{R}\right)^{2}}} p\left(\xi^{z}\right) d \xi^{z} \\
= & -\frac{h_{p} F_{R}}{2}\left\{\frac{\sin \left[2 \pi t \sqrt{\left(h_{p} F_{R}\right)^{2}-\left(\Gamma F_{0}\right)^{2}}\right]}{\sqrt{\left(h_{p} F_{R}\right)^{2}-\left(\Gamma F_{0}\right)^{2}}}-2 \pi \Gamma F_{0} \int_{0}^{t} J_{0}\left(2 \pi h_{p} F_{R} u\right) \frac{\sin \left[2 \pi(t-u) \sqrt{\left(h_{p} F_{R}\right)^{2}-\left(\Gamma F_{0}\right)^{2}}\right]}{\sqrt{\left(h_{p} F_{R}\right)^{2}-\left(\Gamma F_{0}\right)^{2}}} d u\right\}, \\
\overline{\left\langle S^{z}(t)\right\rangle}= & \frac{1}{2} \int_{-\infty}^{+\infty} \frac{\left(F_{0} \xi^{z}\right)^{2}+\left(h_{p} F_{R}\right)^{2} \cos \left[2 \pi t \sqrt{\left(F_{0} \xi^{z}\right)^{2}+\left(h_{p} F_{R}\right)^{2}}\right]}{\left(F_{0} \xi^{z}\right)^{2}+\left(h_{p} F_{R}\right)^{2}} p\left(\xi^{z}\right) d \xi^{z} \\
= & \frac{1}{2}\left\{\frac{-\left(\Gamma F_{0}\right)^{2}+\left(h_{p} F_{R}\right)^{2} \cos \left[2 \pi t \sqrt{\left(h_{p} F_{R}\right)^{2}-\left(\Gamma F_{0}\right)^{2}}\right]}{\left(h_{p} F_{R}\right)^{2}-\left(\Gamma F_{0}\right)^{2}}\right. \\
& \left.+2 \pi \Gamma F_{0}\left(h_{p} F_{R}\right)^{2} \int_{0}^{t} J_{0}\left(2 \pi h_{p} F_{R} u\right) \frac{1-\cos \left[2 \pi(t-u) \sqrt{\left(h_{p} F_{R}\right)^{2}-\left(\Gamma F_{0}\right)^{2}}\right]}{\left(h_{p} F_{R}\right)^{2}-\left(\Gamma F_{0}\right)^{2}} d u\right\} .
\end{aligned}
$$


In the case wherein, initially, all the spins are aligned along the $x$-axis, we find

$$
\begin{aligned}
\overline{\left\langle S^{x}(t)\right\rangle}= & \frac{1}{2} \int_{-\infty}^{+\infty} \frac{\left(h_{p} F_{R}\right)^{2}+\left(F_{0} \xi^{z}\right)^{2} \cos \left[2 \pi t \sqrt{\left(F_{0} \xi^{z}\right)^{2}+\left(h_{p} F_{R}\right)^{2}}\right]}{\left(F_{0} \xi^{z}\right)^{2}+\left(h_{p} F_{R}\right)^{2}} p\left(\xi^{z}\right) d \xi^{z} \\
= & \frac{1}{2}\left\{\frac{\left(h_{p} F_{R}\right)^{2}-\left(\Gamma F_{0}\right)^{2} \cos \left[2 \pi t \sqrt{\left(h_{p} F_{R}\right)^{2}-\left(\Gamma F_{0}\right)^{2}}\right]}{\left(h_{p} F_{R}\right)^{2}-\left(\Gamma F_{0}\right)^{2}}-2 \pi \frac{\Gamma F_{0}\left(h_{p} F_{R}\right)^{2}}{\left(h_{p} F_{R}\right)^{2}-\left(\Gamma F_{0}\right)^{2}} \int_{0}^{t} J_{0}\left(2 \pi h_{p} F_{R} u\right) d u\right. \\
& \left.+2 \pi \frac{\left(\Gamma F_{0}\right)^{3}}{\left(h_{p} F_{R}\right)^{2}-\left(\Gamma F_{0}\right)^{2}} \int_{0}^{t} J_{0}\left(2 \pi h_{p} F_{R} u\right) \cos \left[2 \pi(t-u) \sqrt{\left(h_{p} F_{R}\right)^{2}-\left(\Gamma F_{0}\right)^{2}}\right] d u\right\} .
\end{aligned}
$$

Recall that we calculate the transverse magnetization for the case wherein, initially, all spins are aligned along the $x$-axis. In order to obtain the expressions in terms of elementary functions, we have ignored the cutoff of the Lorentzian distribution. We can check that for $\Gamma=0$, Eqs. (20) and (21) reduce to

$$
\overline{\left\langle S^{z}(t)\right\rangle}=\frac{1}{2} \cos \Omega_{R} t, \quad \overline{\left\langle S^{x}(t)\right\rangle}=\frac{1}{2},
$$

while for $h_{p}=0$, we find

$$
\overline{\left\langle S^{z}(t)\right\rangle}=\frac{1}{2}, \quad \overline{\left\langle S^{x}(t)\right\rangle}=\frac{1}{2} e^{-2 \pi t \Gamma F_{0}},
$$

in agreement with the expressions that can be derived directly, without any averaging procedure. From Eq. (23), it follows that $1 / T_{2}=2 \pi \Gamma F_{0}$. For finite $\Gamma$, Rabi oscillations are present only if $h_{p} F_{R}>\Gamma F_{0}$ in both longitudinal and transverse cases.

In Fig. 8 (left), we present a typical result for the time dependence of the longitudinal magnetization with $g^{z}$-factor distribution (only), suggesting that the time-averaged longitudinal magnetization is nonzero, in concert with the analytical expressions

$$
\begin{aligned}
& \lim _{T \rightarrow \infty} \frac{1}{T} \int_{0}^{T} \overline{\left\langle S^{x}(t)\right\rangle} d t=0 \\
& \lim _{T \rightarrow \infty} \frac{1}{T} \int_{0}^{T} \overline{\left\langle S^{y}(t)\right\rangle} d t=0 \\
& \lim _{T \rightarrow \infty} \frac{1}{T} \int_{0}^{T} \overline{\left\langle S^{z}(t)\right\rangle} d t=\frac{1}{2} \frac{\Gamma F_{0}}{h_{p} F_{R}+\Gamma F_{0}} .
\end{aligned}
$$

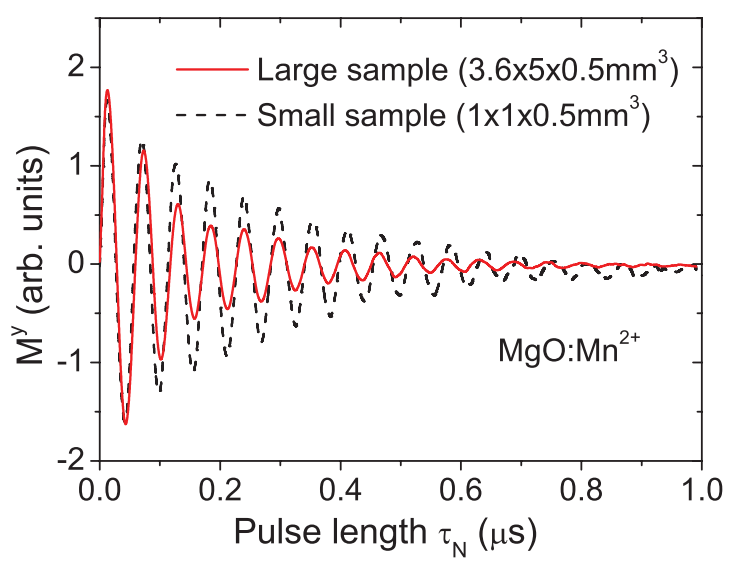

FIG. 7. (Color online) Rabi oscillations of MgO: $\mathrm{Mn}^{2+}(0.001 \%)$ for two sample sizes. Measurements were carried out at room temperature.
The reason for this positive offset is simple: Any nonzero field in the $z$ direction tilts the plane of the Rabi oscillations away from the $(y, z)$ plane, introducing a small precession about the tilted axis superimposed on the Rabi nutation, leading to a positive long-time average. This nonzero offset effect is significant because, as we will see later, it is a unique signature of the presence of random fluctuations in the $g^{z}$-factor or, equivalently, of the inhomogeneity of the static magnetic field. We emphasize that this nonzero offset is due to randomness and not due to dissipation, as this paper considers the case of $T_{1}=\infty$ only.

Similarly, in the case that all spins are initially along the $x$ direction, the long-time average of the transverse magnetization is given by

$$
\lim _{T \rightarrow \infty} \frac{1}{T} \int_{0}^{T} \overline{\left\langle S^{x}(t)\right\rangle} d t=\frac{1}{2} \frac{h_{p} F_{R}}{h_{p} F_{R}+\Gamma F_{0}},
$$

with the long-time averages of the two other components being zero. Unlike in the case of the longitudinal magnetization, in the regime where the transverse magnetization shows oscillations $\left(h_{p} F_{R}>\Gamma F_{0}\right)$, the transverse magnetization reaches its asymptotic value Eq. (25) already after a few oscillations (data not shown).

From Eq. (20), it is clear that we can not expect the amplitude of the Rabi oscillations to decay exponentially in a strict sense. Nevertheless, the data fit well to a function of the form $\left(a+b e^{-c_{\mathrm{R}} t} \cos 2 \pi f t\right) / 2$. The decay rate $c_{\mathrm{R}}$, shown in Fig. 8 (right), decreases with increasing microwave amplitude $h_{p}$. It seems to diverge when $h_{p} \rightarrow 0$, but this is never observed in experiment. This decrease is a second characteristic feature of the presence of random fluctuations in the $g^{z}$-factor or, equivalently, of the inhomogeneity of the static magnetic field.

\section{Randomness in $g^{x}$ and $g^{y}$ : Noninteracting spins}

In this case, the Hamiltonian is given by Eq. (5) with $\zeta_{j}=$ $\xi^{z}=D_{0}=0$ and we have

$$
\begin{aligned}
\overline{\left\langle S^{z}(t)\right\rangle}= & \frac{1}{2} \int_{-\infty}^{+\infty} \cos \left\{\Omega_{R} t\left[1+\left(\xi^{x}+\xi^{y}\right) / 2\right]\right\} \\
& \times p\left(\xi^{x}\right) p\left(\xi^{y}\right) d \xi^{x} d \xi^{y} .
\end{aligned}
$$

Taking the cutoff $\xi_{0}$ to be infinity, we obtain

$$
\begin{aligned}
\overline{\left\langle S^{z}(t)\right\rangle} & =\frac{1}{2 \pi} \int_{-\infty}^{+\infty} \cos \left[\Omega_{R} t(1+\zeta)\right] \frac{\Gamma}{\zeta^{2}+\Gamma^{2}} d \zeta \\
& =\frac{1}{2} e^{-\Omega_{R} \Gamma t} \cos \Omega_{R} t .
\end{aligned}
$$



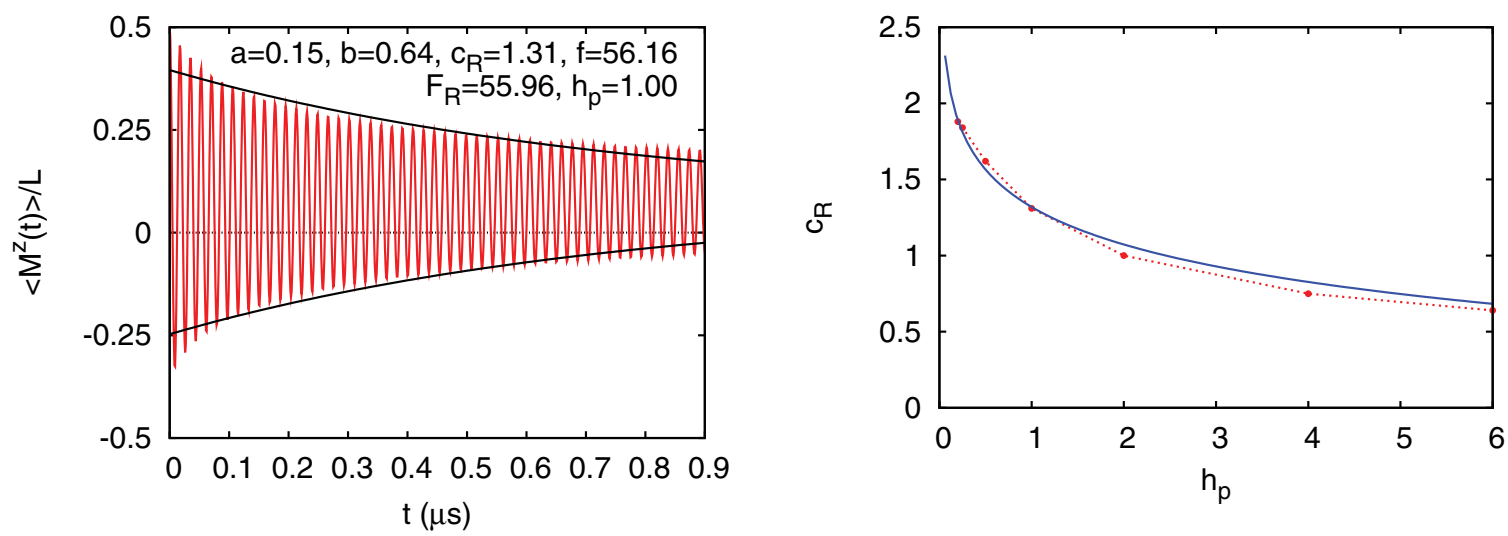

FIG. 8. (Color online) Left: Time evolution of the longitudinal magnetization as obtained by numerical evaluation of Eq. (20) (or the solution of the TDSE for $D_{0}=\xi^{x}=\xi^{y}=\zeta=0$ for $\left.\Gamma=0.001\right)$, that is, for the case wherein there are random fluctuations in the $g^{z}$-factor only. The solid line represents the envelope $\left(a \pm b e^{-c_{\mathrm{R}} t}\right) / 2$ of the function $\left(a+b e^{-c_{\mathrm{R}} t} \cos 2 \pi f t\right) / 2$ that was fitted to the data. Right: The decay rate $c_{\mathrm{R}}$ as a function of $h_{p}$, obtained by fitting $\left(a+b e^{-c_{\mathrm{R}} t} \cos 2 \pi f t\right) / 2$ to the time-dependent data. The solid line shows that the function $a^{\prime} \ln \left(h_{p}\right)+b^{\prime}$ with $a^{\prime}=-0.35$ and $b^{\prime}=1.32$ fits the data reasonably well. The dashed line connecting the bullets is a guide to the eye only.

Thus, we conclude that if there is randomness in $g^{x}$ and $g^{y}$ only, the Rabi oscillations will decay exponentially with a rate proportional to $\Omega_{R}=2 \pi h_{p} F_{R}$. In the absence of the microwave field, the transverse magnetization is a constant of motion and hence $T_{2}=\infty$. Summarizing, in the presence of randomness in $g^{x}$ and $g^{y}$ only and in the absence of dipole-dipole interactions, we have

$$
1 / T_{R}=\Gamma \Omega_{R}>1 / T_{2}=0,
$$

showing that the decay rate of the Rabi oscillations increases linearly with the microwave amplitude $h_{p}$. In fact, Eq. (28) is the same as Eq. (18) with $\gamma$ replaced by $\Gamma$. Thus, we conclude that randomness in $g^{x}$ and $g^{y}$ has the same effect as randomness in the amplitude of the microwave field: The Rabi oscillations decay exponentially, with a decay rate that increases linearly with $\Omega_{R}=2 \pi h_{p} F_{R}$. In both cases, decoherence results from a loss of phase of superposed radiation emitted by spins in nutation leading, as a consequence, to energy transfer from the spin bath to the electromagnetic bath. Clearly enough, such dissipation does not involve the usual relaxation time $T_{1}$ due to dissipation by phonons. This case is very different from the one of, e.g., superconducting qubits where decoherence is dominated by $T_{1}$ process, as shown for example in Ref. 26.

\section{Randomness in $g^{x}, g^{y}$, and $g^{z}$ : Noninteracting spins}

In this case, the Hamiltonian is given by Eq. (5) with $\zeta_{j}=$ $D_{0}=0$. In Fig. 9 (top), we present a typical result for the time dependence of the longitudinal magnetization. It is seen that the time-averaged longitudinal magnetization is nonzero, signaling the presence of fluctuations in $g^{z}$ (see Sec. III C 1). Also, clearly visible is the increase of the decay rate $c_{R}$ of the Rabi oscillations with increasing microwave amplitude $h_{p}$, a signal of the presence of fluctuations in $\left(g^{x}, g^{y}\right)$ (see Sec. III C 2). Note that there is no obvious relation between the decay rate of the transverse magnetization $\left[c_{2} \approx 60\right.$, see
Fig. 9 (bottom left)] and the values of the decay rate $c_{\mathrm{R}}$ at the smallest values of $h_{p}$ shown in Fig. 9 (bottom right).

From the results of Secs. III C 1 and III C 2, we may expect that the decay rate $c_{\mathrm{R}}$ shows a crossover from the regime in which the fluctuations on $g^{z}$ dominate $\left(c_{\mathrm{R}}\right.$ decreases with increasing $h_{p}$ ) and a regime in which the fluctuations on $\left(g^{x}, g^{y}\right)$ dominate $\left(c_{\mathrm{R}}\right.$ increases linearly with $\left.h_{p}\right)$. This is borne out by the data presented in Fig. 9 (bottom right) where we show the combined effect of the two different sources of decoherence, with the widths of the Lorenztian distributions for the longitudinal $\left(g^{z}, \Gamma^{z}\right)$ and transverse $\left[\left(g^{x}, g^{y}\right), \Gamma^{x}=\Gamma^{y}\right]$ fluctuations being varied independently.

\section{Experimental results: $\mathrm{MgO}: \mathrm{Mn}^{2+}$}

The combined effect of a distribution in the $g$-factors and inhomogeneities in the microwave amplitude are shown in experiments performed on single-crystalline films of $\mathrm{MgO}: \mathrm{Mn}^{2+}$ (see Fig. 10 where the measured Rabi decay time is plotted versus the Rabi frequency). The $\mathrm{Mn}^{2+}$ dilution is such that dipolar interactions are negligible. Due to weak but sizable distributions of $\mathrm{Mn}^{2+}$ local environments, we expect non-negligible and similar distributions of the three $g$-factor components. For small microwave amplitudes, the distribution in the $g^{z}$-factor gives the dominant, nearly constant contribution to the Rabi decay time, which compares well with Fig. 9 (bottom right). As the microwave amplitude increases, the inhomogeneities associated with transverse components take over and $1 / T_{R}$ increases linearly on the log-log scale. Note that the slope of one-half differs from the slope one that we have for the model considered in this paper. This is because of the peculiarity of the experimental system where nutation takes place coherently over five equidistant levels of the material, an aspect that will be considered in the future. At present, we are interested in showing that the departure from the $1 / T_{R}$ plateau takes place more rapidly with the larger sample as expected when the effect of microwave inhomogeneities dominates over the one of $g$-factor distributions. 

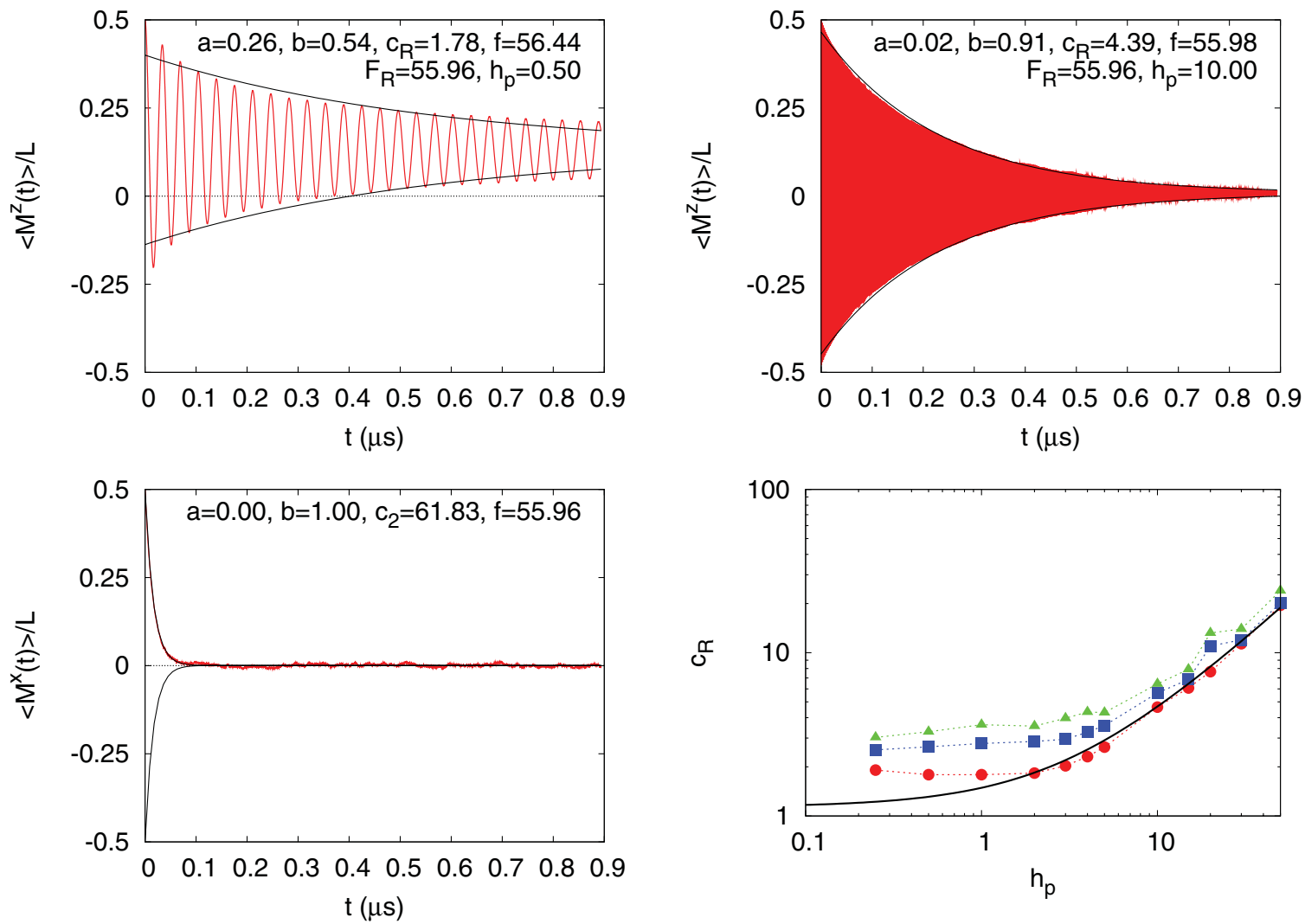

FIG. 9. (Color online) Top left: Time evolution of the longitudinal magnetization as obtained by numerical solution of the TDSE [see Eq. (11)] for the case wherein there are random fluctuations in all three $g$-factors only $\left(\Gamma=\Gamma^{x}=\Gamma^{y}=\Gamma^{z}=0.001\right.$ and $\left.\zeta=D_{0}=0\right)$. The solid line represents the envelope $\left(a \pm b e^{-c_{\mathrm{R}} t}\right) / 2$ of the function $\left(a+b e^{-c_{\mathrm{R}} t} \cos 2 \pi f t\right) / 2$ that was fitted to the data. Top right: Same as top right, except that $h_{p}=10$ instead of $h_{p}=0.5$. Bottom left: Transverse magnetization in the absence of the microwave field $\left(h_{p}=0\right)$. The decay rate $c_{2}=60.19$ is in excellent agreement with the analytical result $c_{\mathrm{R}}=2 \pi \Gamma F_{0}=60.95$ predicted by Eq. (23). The solid line represents the function $\left(a+b e^{-c_{2} t}\right) / 2$ that was fitted to the data. Bottom right: The inverse relaxation time $c_{\mathrm{R}}$ as a function of the microwave amplitude $h_{p}$ for $\Gamma^{x}=\Gamma^{y}=\Gamma^{x}=0.001$ and $\Gamma^{z}=0.001$ (bullets), $\Gamma^{z}=0.002$ (squares), $\Gamma^{z}=0.003$ (triangles). The solid line represents the linear fit to the $\Gamma^{z}=0.001$ data. The dashed lines are guides to the eye only. The number of spins in these calculations is 10000 .

\section{Dipolar-coupled spins}

In Fig. 11 (top and middle), we present simulation results for systems of 26 spins with dipole-dipole interaction (with

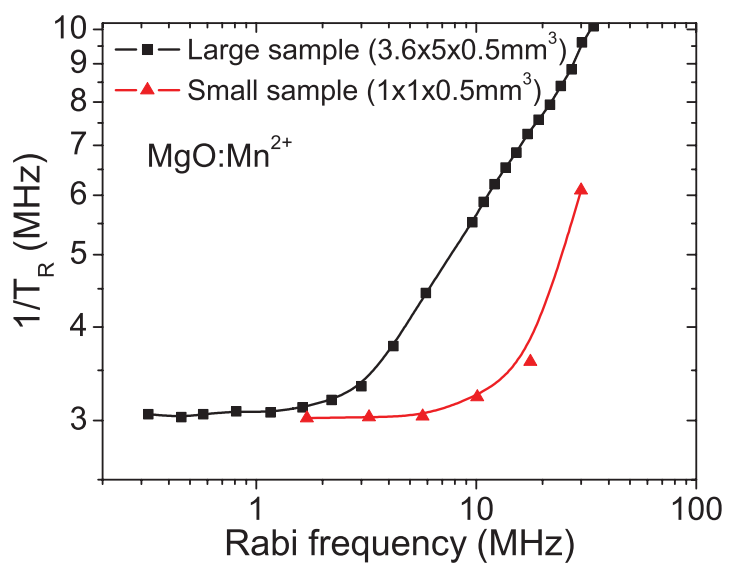

FIG. 10. (Color online) Decay time of the Rabi oscillations of $\mathrm{MgO}: \mathrm{Mn}^{2+}(0.001 \%)$ as a function of the microwave field amplitude (Rabi frequency $\Omega_{R}$ ) for two samples of different sizes. Measurements were carried out at room temperature. different concentrations $n$ ), with random fluctuations in the three $g$-factors and uniform microwave field amplitude. These results are obtained by averaging over 10 different realizations, meaning 10 different distributions of the 26 spins on the lattice. The striking signature of the presence of fluctuations in $g^{z}$, namely, the nonzero long-time average of the longitudinal magnetization, remains untouched by the effects of the dipolar interactions. For the values of $h_{p}$ shown in Fig. 11 (top left to middle right), the dependence of the decay rate $c_{\mathrm{R}}$ is essentially the same as if the dipolar interactions were absent [see Fig 9 (bottom right)]. For large $h_{p}$ (data not shown), the decay rate $c_{\mathrm{R}}$ linearly increases with $h_{p}$. Comparing Fig. 11 (bottom left) with Fig. 11 (bottom right), it follows that the value of the decay rate of the transverse magnetization is nearly independent of the concentration, hence, can not be attributed to the presence of dipolar interactions, but is mainly due to the presence of fluctuations in $g^{z}$.

\section{Randomness in the $g$-factors and the microwave amplitude}

\section{Noninteracting spins}

In Fig. 12, we present a few representative results for the case wherein there are random fluctuations in both the 

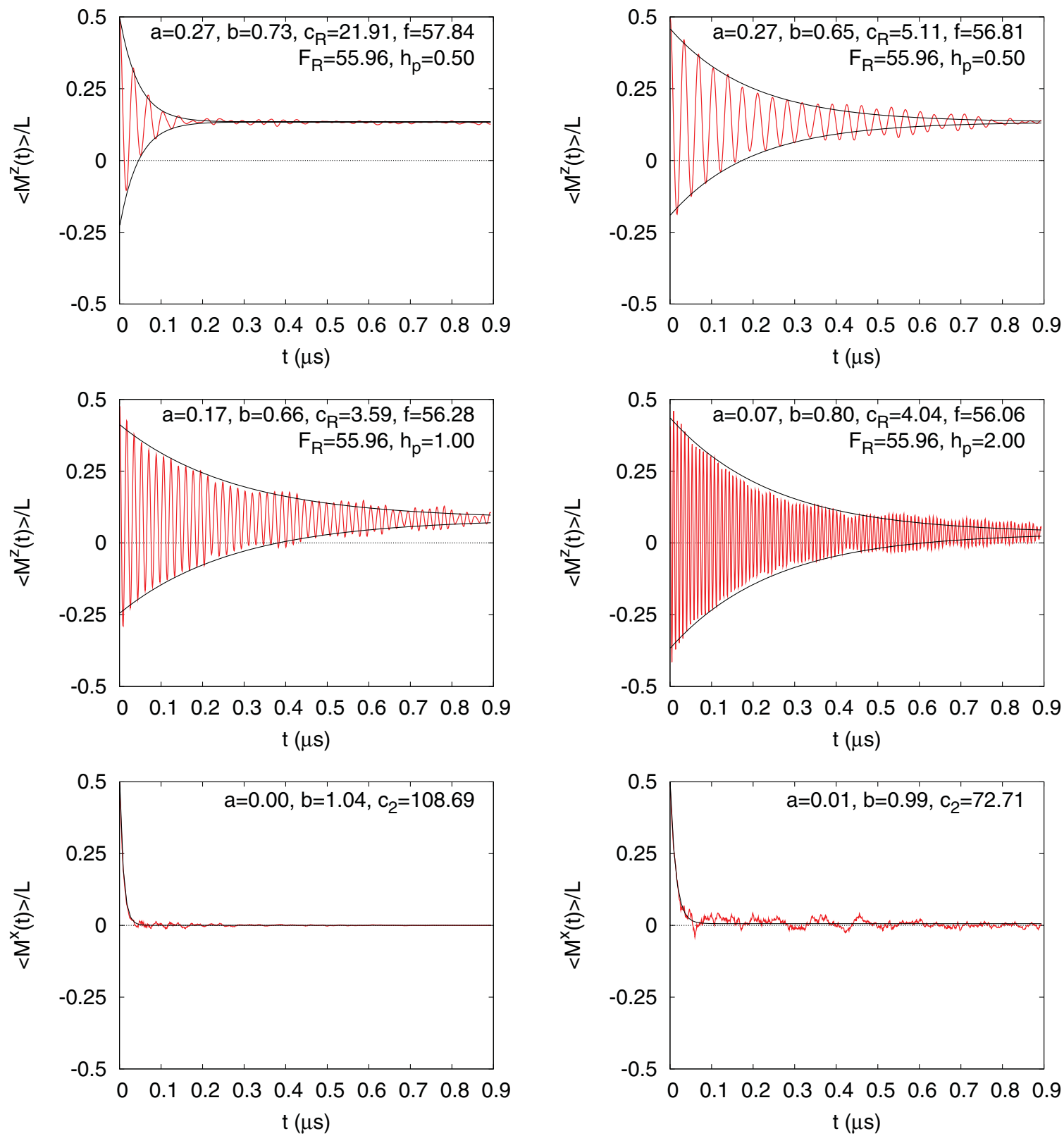

FIG. 11. (Color online) The Rabi oscillations of the longitudinal magnetization as obtained by solving the TDSE with the Hamiltonian (5) for 26 spins that interact via dipole-dipole interaction, for different concentrations $n$, with random fluctuations in the three $g$-factors $(\Gamma=0.001)$ and without random fluctuations in the microwave amplitude $(\gamma=0)$. Top left: $n=10^{-3}$. Top right to middle right: $n=10^{-4}$. The solid line represents the envelope $\left(a \pm b e^{-c_{\mathrm{R}} t}\right) / 2$ of the function $\left(a+b e^{-c_{\mathrm{R}} t} \cos 2 \pi f t\right) / 2$ that was fitted to the data. Bottom: Time evolution of the transverse magnetization for $n=10^{-3}$ (left) and $n=10^{-4}$ (right). The solid line represents the function $\left(a+b e^{-c_{2} t}\right) / 2$ that was fitted to the data.

microwave amplitude and in the $g$-factors, as obtained by solving the TDSE for the Hamiltonian (5) with $D_{0}=0$. In essence, the results are very similar to those of the case where there are fluctuations in all three $g$-factors only. This is easy to understand from Eq. (5): Fluctuations in $\left(g^{x}, g^{y}\right)$ or (exclusive) in the microwave amplitude have the same effect on the decay of the Rabi oscillations. With both types of fluctuations present, our numerical results show that this contribution does not significantly alter the dependence of $c_{\mathrm{R}}$ on $h_{p}$.

As before, the presence of fluctuations in $g^{z}$ (see Sec. III C 1) is signaled by the time-averaged longitudinal magnetization being nonzero and by a contribution to the decay rate $c_{2}$ of the transverse magnetization, which is in excellent agreement with the analytical result $c_{2}=2 \pi \Gamma F_{0}$ predicted by Eq. (23) (data not shown). Thus, in this case, we obviously have $c_{2}>c_{\mathrm{R}}$, which is the same as $T_{R}>T_{2}$ where $T_{2}$ is reduced by the fluctuations in $g^{z}$.

\section{Dipolar-coupled spins}

In Fig. 13, we present simulation results for systems of 12 spins with dipole-dipole interaction, as obtained by averaging the solution of the TDSE over 100 different distributions of the 12 spins on the lattice, for the case that there are random 

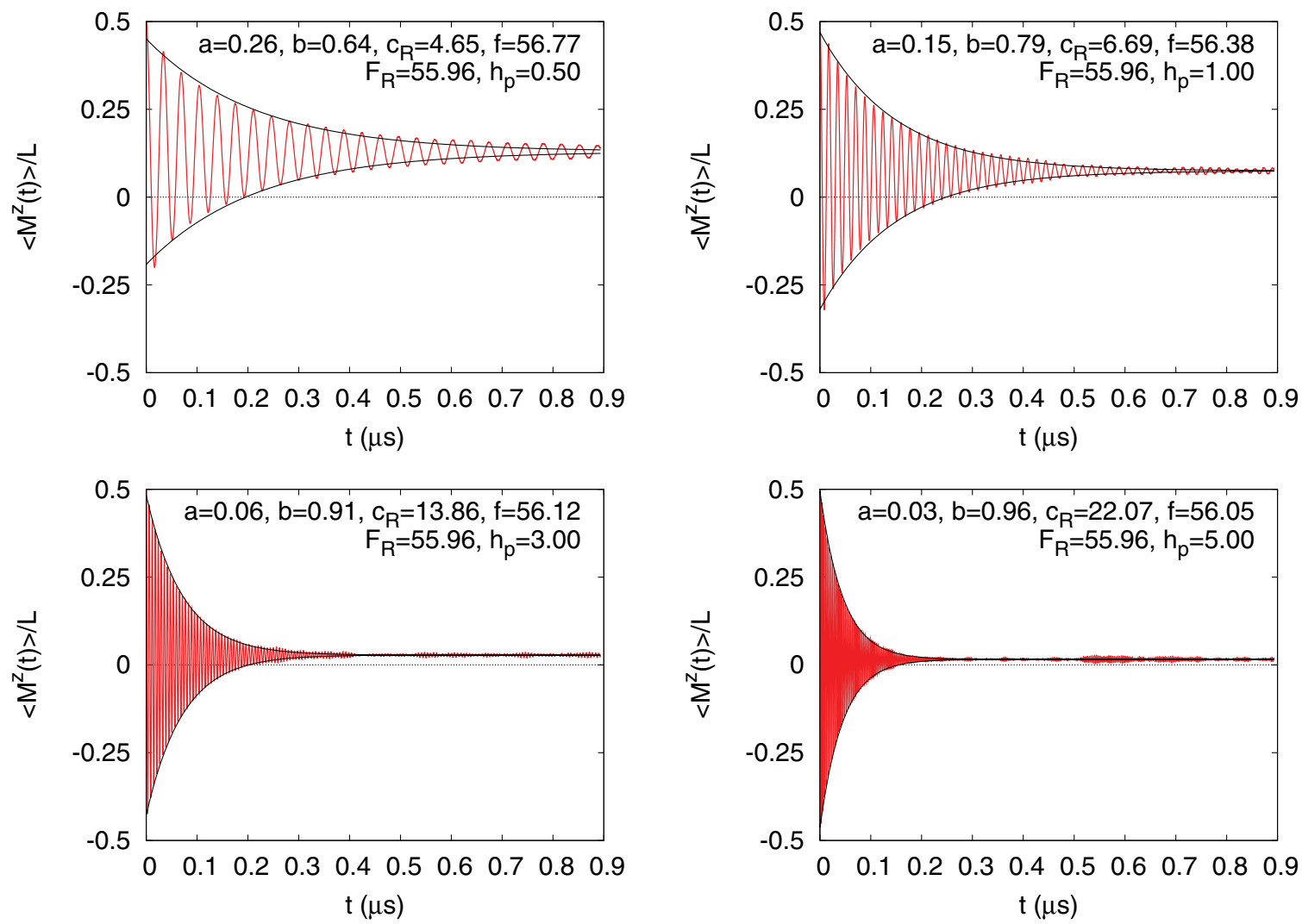

FIG. 12. (Color online) The Rabi oscillations as obtained from the numerical solution of the TDSE [see Eq. (11)] for $D_{0}=0, \gamma=0.01$, and $\Gamma=0.001$, that is, for the case wherein there are random fluctuations in both the microwave field and in the $g$-factors. The solid line represents the envelope $\left(a \pm b e^{-c_{\mathrm{R}} t}\right) / 2$ of the function $\left(a+b e^{-c_{\mathrm{R}} t} \cos 2 \pi f t\right) / 2$ that was fitted to the data. The number of spins in these calculations is 10000 .

fluctuations in the microwave amplitude and in all three $g$-factors. The four upper panels of Fig. 13 show results for the longitudinal magnetization. The decay of the longitudinal magnetization is exponential to good approximation. The signature of the presence of fluctuations in $g^{z}$, namely, the nonzero long-time average of the longitudinal magnetization is clearly visible. For the values of $h_{p}$ shown in Fig. 13 (bottom left), the linear dependence of the decay rate $c_{\mathrm{R}}$ is essentially the same as if the dipolar interactions were absent [see Fig. 9 (bottom right)].

A linear fit to the data of $c_{\mathrm{R}}$ yields $\lim _{h_{p} \rightarrow 0} c_{\mathrm{R}} \approx 4.43$. This value should be contrasted with the result $c_{2} \approx 63.74$ for the transverse magnetization in the absence of microwaves $\left(h_{p}=0\right)$ [see Fig. 13 (bottom right)]. Such a large $c_{2}$ (small $T_{2}$ ) resulting from both dipolar interactions and fluctuations on all $g$-factors is effectively caused by the effect of $g^{z}$ fluctuations, in concert with the results shown in Fig. 11 (bottom) that demonstrate that the concentration dependence is weak, implying that the effect of the dipolar interactions is small compared to that of the presence of fluctuations in $g^{z}$.

According to theory, the total decay rate of the transverse magnetization is the sum of the decay rates due to the dipolar interactions only and the combined decay rate due to field inhomogeneities only. From Fig. 5, the former is given by $c_{2} \approx 3.90$. In the absence of dipolar interactions, the latter is given by $c_{2}=2 \pi \Gamma F_{0}=60.95$ (see Sec. III C 3 and Fig. 9, yielding $c_{2} \approx 60.19$ for $\gamma=0$ ). Therefore, we have $c_{2}^{\text {total }} \approx$ 64.85 , in very good agreement with the value $c_{2}=63.74$ extracted from the simulation (see bottom right panel of Fig. 13).

\section{PHENOMENOLOGICAL MODEL}

The simulations of the dipolar-coupled spin systems are rather expensive in terms of computational resources. For instance, one simulation of a single realization of a 26spin system takes about 20 hours, using 512 CPUs on an IBM BlueGene/P. Such relatively expensive simulations are necessary to disentangle the various mechanisms that may cause decoherence but are not useful as a daily tool for analyzing experiments. Therefore, it is of interest to examine the possibility as to whether a simple phenomenological model can capture the essence of the physics of the full microscopic model. Based on our results, presented in Sec. III, we propose to use a single-spin model to which we artificially add a dephasing and relaxation mechanism.

Specifically, we propose that the Heisenberg equation of motion (in the rotating frame) of the expectation values of the 

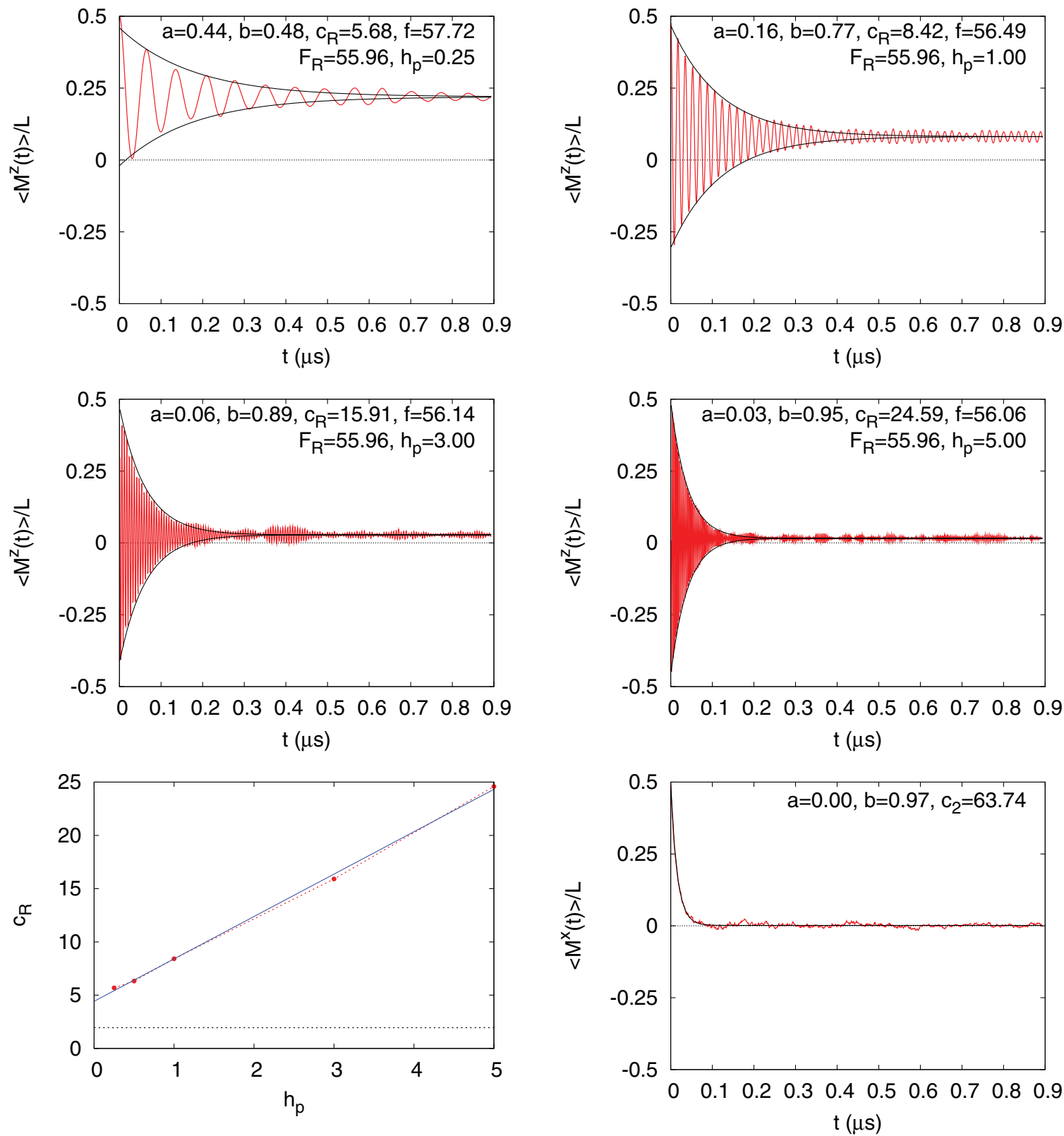

FIG. 13. (Color online) Simulation results as obtained by solving the TDSE for the Hamiltonian (5) for 12 spins (concentration $n=10^{-4}$ ) that interact via dipole-dipole coupling, with random fluctuations in the $g$-factors $(\Gamma=0.001)$, and with random fluctuations in the microwave amplitude $(\gamma=0.01)$. Top left to middle right: Longitudinal magnetization showing Rabi oscillations. The solid line represents the envelope $\left(a \pm b e^{-c_{\mathrm{R}} t}\right) / 2$ of the function $\left(a+b e^{-c_{\mathrm{R}} t} \cos 2 \pi f t\right) / 2$ that was fitted to the data. Bottom left: Bullets show the inverse relaxation time $c_{\mathrm{R}}=1 / T_{R}$ as a function of the microwave amplitude $h_{p}$. The dashed line connecting the bullets is a guide to the eye only. A linear fit to the simulation data yields $c_{\mathrm{R}}=1 / T_{R} \approx 3.98 h_{p}+4.43$ and is shown by the solid line. The horizontal line represents the value of $1 / 2 T_{2} \approx 1.95$, estimated from the data of the transverse magnetization in the absence of random fluctuations in the $g$-factors and on the microwave amplitude (see Fig. 5). Bottom right: Transverse magnetization in the absence of the microwave field $\left(h_{p}=0\right)$. The solid line represents the function $\left(a+b e^{-c_{2} t}\right) / 2$ that was fitted to the data. The decay rate $c_{2}$ contains contributions from the dipolar interactions and, most importantly, from the random fluctuations in $g^{z}$.

spin components is modified according to

$$
\frac{\partial}{\partial t}\langle\mathbf{S}(t)\rangle=\left(\begin{array}{ccc}
-1 / T_{2} & 2 \pi \xi^{z} F_{0} & 0 \\
-2 \pi \xi^{z} F_{0} & -1 / T_{2} & \pi h_{p}(1+\zeta)\left(2+\xi^{x}+\xi^{y}\right) F_{R} \\
0 & -\pi h_{p}(1+\zeta)\left(2+\xi^{x}+\xi^{y}\right) F_{R} & -1 / T_{1}
\end{array}\right)\langle\mathbf{S}(t)\rangle
$$


where we adopt the same notation as the one used in Sec. II A. The phenomenological aspect enters in the introduction of the decay times $T_{1}$ and $T_{2}$.

Equation (29) has the same structure as the Bloch equation, but there is a conceptual difference and a practical consequence. The former comes from the introduction of $g$-factor and microwave field amplitude distributions and the latter offers the possibility to calculate numerically the effects of one-spin decoherence to a high degree of accuracy. As we showed in this paper, one-spin decoherence plays an essential role when several qubits act at the same time. It is then natural to start from the well-known equation of motion of a spin $S=1 / 2$, add disorder through distribution probabilities (here of $g$-factors and microwave field amplitude), and average over the solutions. This leads to the exact knowledge of corresponding one-spin decoherence, namely, to Eq. (29) without the $T_{1}$ and $T_{2}$ terms. If we now want to make a link with the Bloch equations, we have just to add the phenomenological damping times $T_{1}$ and $T_{2}$ as it is done in the original Bloch equations. The difference between Eq. (29) and the original Bloch equations is that, in the latter, $T_{1}$ and $T_{2}$ include all damping contributions, i.e., many-spin and one-spin damping, whereas in the former, $T_{1}$ and $T_{2}$ include manyspins damping only, with one-spin damping being calculated exactly.

Before assessing the usefulness of Eq. (29) by comparing its results to the numerical solution of the TDSE of the interacting spin system, it is instructive to analyze the case $\xi^{x}=\xi^{y}=$ $\xi^{z}=\zeta=T_{1}=0$. Then, the solution of Eq. (29) reads as

$$
\begin{aligned}
& \left\langle\mathbf{S}^{x}(t)\right\rangle=e^{-t / T_{2}}\left\langle\mathbf{S}^{x}(0)\right\rangle, \\
& \left\langle\mathbf{S}^{z}(t)\right\rangle=e^{-t / 2 T_{2}} \cos \left(2 \pi h_{p} F_{R} \sqrt{1-\left(1 / 4 \pi h_{p} F_{R} T_{2}\right)^{2}}\right)\left\langle\mathbf{S}^{z}(0)\right\rangle,
\end{aligned}
$$

where, for simplicity, we have assumed that $\left\langle\mathbf{S}^{y}(0)\right\rangle=0$. From Eq. (30), it follows that the transverse and longitudinal magnetization decays exponentially with a relaxation time $T_{2}$ and $2 T_{2}$, respectively. In other words, in the absence of randomness and for $T_{1}=0$, Eq. (29) predicts a factor of 2 between the relaxation time of the Rabi oscillations and the relaxation time of the transverse magnetization, in qualitative (and almost quantitative) agreement with our simulation results of dipolar-coupled spin-1/2 systems with randomness. Thus, model Eq. (29) may give a simple explanation as to why in our simulations we find that extrapolation of $c_{\mathrm{R}}$ to $h_{p}=0$ gives, in the presence of dipolar interactions, precisely $c_{2} / 2$ if there is no distribution of $g$-factors $\left(D_{0} \neq 0, \Gamma=0\right)$ and a value larger than $c_{2}$ if there is a distribution of $g$-factors $(\Gamma>0)$.

If we set $\xi^{x}=\xi^{y}=\xi^{z}=\zeta=0$, which in principle we should do if we strictly adopt the Bloch-equations approach, we can never recover the linear dependence of the decay rate $1 / T_{R}$ on the microwave amplitude $h_{p}$. However, if we average over the $\xi$ 's and/or $\zeta$ and set $T_{2}=\infty$, the results are the same as those obtained from the direct solution of the TDSE of the spin- $1 / 2$ system.

In Appendix B, we give a simple, robust, unconditionally stable algorithm ${ }^{27}$ to solve Eq. (29). In Fig. 14, we present some representative results. We used the same parameters for $\Gamma, \gamma$, and $h_{p}$ and changed the phenomenological parameter $T_{2}$ until we found a fair match with the data of the corresponding interacting system. Taking into account that we did not attempt to make a best fit to these data, the agreement is excellent. In both cases shown in Fig. 14 (and in many others cases not shown), this simple procedure seems to work quite well. This suggests that the simple model Eq. (29) may be very useful for the analysis of experimental data, including the effects of the pulse sequence and pulse shapes, effects that are rather expensive to analyze using the large-scale simulation approach adopted in this paper.

\section{SUMMARY AND OUTLOOK}

The main results of this paper may be summarized as follows:

(i) The noninteracting spin model can account for the $\Omega_{R}$ dependence of the decay of the Rabi oscillations if we introduce randomness in the $g$-factors (all three) and/or in the amplitude of the microwave field. In the case of $g^{z}$ randomness, the long-time average of the longitudinal
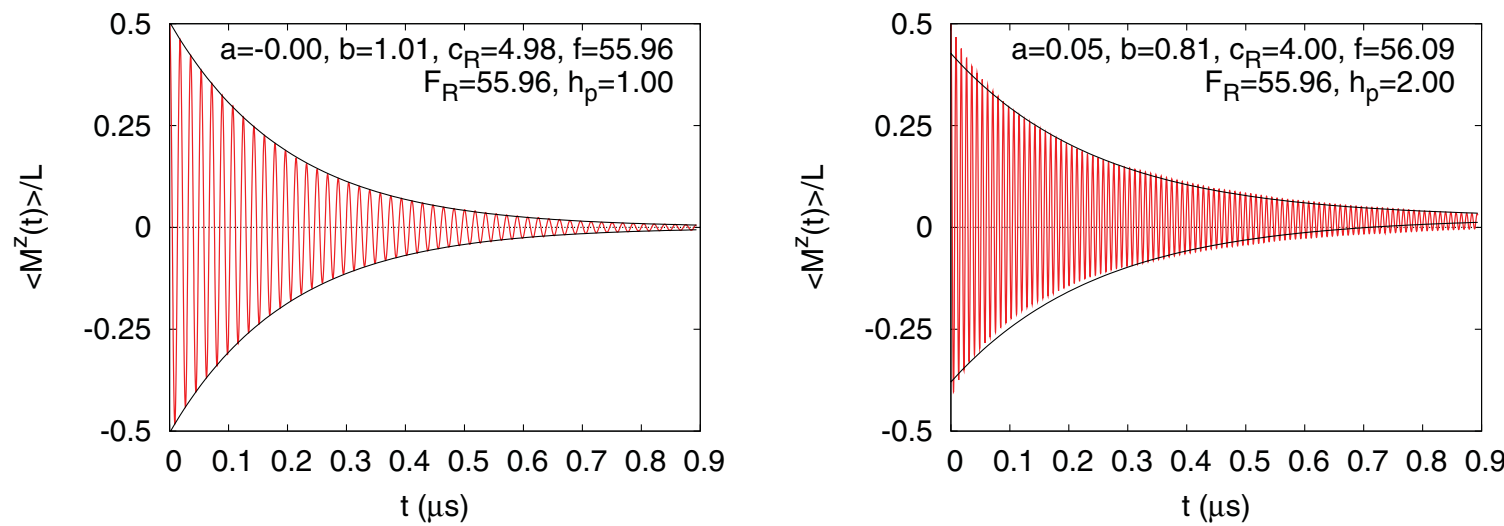

FIG. 14. (Color online) The Rabi oscillations of the longitudinal magnetization as obtained from the numerical solution of the phenomenological model Eq. (29) with $T_{1}=\infty$, using 10000 realizations of the random variables $\xi^{x}, \xi^{y}, \xi^{z}$, and $\zeta$. Left: $\Gamma=0, \gamma=0.01$, $1 / T_{1}=0$, and $T_{2}=3.0$ [compare with Fig. 5 (top right)]. Right: $\Gamma=0.001, \gamma=0,1 / T_{1}=0$, and $T_{2}=3.6$ [compare with Fig. 11 (middle right)]. The solid line represents the envelope $\left(a \pm b e^{-c_{\mathrm{R}} t}\right) / 2$ of the function $\left(a+b e^{-c_{\mathrm{R}} t} \cos 2 \pi f t\right) / 2$ that was fitted to the data. 
magnetization deviates from zero. This deviation increases as the Rabi frequency decreases and reaches its maximum (1/2) when $h_{p} F_{R} / \Gamma F_{0} \rightarrow 0$. The effect of the $g^{z}$ distribution on the value of $c_{\mathrm{R}}$ at zero microwave field $\left(h_{p}=0\right)$ is simply related to the value of $\left\langle M^{z}(t=\infty)\right\rangle$, suggesting that this decoherence effect comes from the combination of different spin precessions about the $z$ axes and the nutational motion of spins.

(ii) The dipolar-coupled spin system without randomness in all three $g$-factors and without randomness in the amplitude of the microwave field can not account for the $\Omega_{R}$ dependence of the Rabi oscillation decay rate, observed in experiment. The decay rate of the Rabi oscillations increases as the concentration of magnetic moments increases, as one naively would expect.

(iii) The dipolar-coupled spin system without randomness in $g^{z}$ but with randomness in the amplitude of the microwave field and/or randomness in $\left(g^{x}, g^{y}\right)$ can account for the $h_{p}$ dependence of the Rabi oscillation decay rate and also for the concentration dependence of this decay rate, just as in the case of noninteracting spins.

(iv) The dipolar-coupled spin system with randomness in all three $g$-factors and with or without randomness in the amplitude of the microwave field can account for the $h_{p}$ dependence of the Rabi oscillation decay rate and also for the concentration dependence of this decay rate. A salient feature of the presence of fluctuations on $g^{z}$ (or, equivalently, on inhomogeneities in the static field) is that the long-time average of the longitudinal magnetization deviates from zero, as in the case of noninteracting spins.

For future work, we want to mention that the effects on the decay of the Rabi oscillations of the measurement by the spin-echo pulses themselves may be studied by the simple phenomenological model described in Sec. IV. Among other aspects, not touched upon in this study, are the case where motional narrowing is important ${ }^{28}$ or where dipolar interactions are strong enough to induce decoherence by magnons, as recently shown in the $\mathrm{Fe}_{8}$ single molecular magnet. ${ }^{29}$ These cases can be treated by the simulation approach adopted in this paper, and we plan to report on the results of such simulations in the near future.

\section{ACKNOWLEDGMENTS}

This work was supported by NCF, The Netherlands (HDR), the Mitsubishi Foundation (SM), and the city of Marseille, Aix-Marseille University (S.B., BQR grant). We thank the multidisciplinary EPR facility of Marseille (PFM Saint Charles) for technical support. Part of the calculations has been performed on JUGENE at JSC under VSR project 4331 .

\section{APPENDIX A: OVERVIEW OF THE MODEL PARAMETERS}

For convenience, we list the parameters of our model:

(i) The Larmor frequency $F_{0}=\omega_{0} / 2 \pi \hbar=9700 \mathrm{MHz}$, which is fixed.

(ii) The Rabi frequency at a microwave amplitude of $1 \mathrm{mT}$ is $F_{R}=55.96 \mathrm{MHz}$, which is fixed.

(iii) The amplitude of the microwave pulse, controlled by the parameter $h_{p}$. By convention, if $h_{p}=1$, a single isolated spin will perform Rabi oscillations with a frequency of $F_{R}=$ $55.96 \mathrm{MHz}$. The Rabi pulsation in the microwave field $h_{p}$ is $\Omega_{R}=2 \pi F_{R} h_{p}$.

(iv) The width $\gamma$ of the Lorentzian distribution of the random fluctuations of the amplitude of the microwave pulse $h_{p}$.

(v) The width $\Gamma$ of the Lorentzian distribution of the random fluctuations of $g^{x}, g^{y}$, and $g^{z}$. Unless mentioned explicitly, we assume that $g^{x}, g^{y}$, and $g^{z}$ share the same distribution.

(vi) The dipole-dipole coupling strength $D_{0}=51.88 \mathrm{GHz}$, which is fixed.

(vii) The concentration $n$ of magnetic impurities on the diamond lattice.

\section{APPENDIX B: NUMERICAL SOLUTION OF THE PHENOMENOLOGICAL MODEL}

As in the case of the Bloch equations, if the relaxation time $T_{1}$ is finite, it is useful to be able to specify both the initial value $\langle\mathbf{S}(t=0)\rangle=\langle\mathbf{S}(0)\rangle$ of the magnetization and its stationary-state value $\langle\mathbf{S}(t=\infty)\rangle=\langle\mathbf{S}\rangle_{0}$. Therefore, we extend Eq. (29) to

$$
\frac{\partial}{\partial t}\langle\mathbf{S}(t)\rangle=\mathbf{A}\langle\mathbf{S}(t)\rangle+\mathbf{b},
$$

where

$$
\mathbf{A}=\left(\begin{array}{lcc}
-1 / T_{2} & 2 \pi \xi^{z} F_{0} & 0 \\
-2 \pi \xi^{z} F_{0} & -1 / T_{2} & \pi h_{p}(1+\zeta)\left(2+\xi^{x}+\xi^{y}\right) F_{R} \\
0 & -\pi h_{p}(1+\zeta)\left(2+\xi^{x}+\xi^{y}\right) F_{R} & -1 / T_{1}
\end{array}\right)
$$

and $\mathbf{b}^{T}=\langle\mathbf{S}\rangle_{0} / T_{1}$. The formal solution of Eq. (B1) reads as

$$
\begin{aligned}
\langle\mathbf{S}(\mathbf{t})\rangle(t) & =e^{t \mathbf{A}}\langle\mathbf{S}(0)\rangle+\int_{0}^{t} e^{u \mathbf{A}} \mathbf{b} d u \\
& =e^{t \mathbf{A}}\langle\mathbf{S}(0)\rangle+\mathbf{A}^{-1}\left(1-e^{t \mathbf{A}}\right) \mathbf{b} .
\end{aligned}
$$


We integrate Eq. (B1), that is, we compute $e^{t \mathbf{A}}$, using the product formula $e^{t \mathbf{A}}=\left(e^{\tau \mathbf{A}_{1} / 2} e^{\tau \mathbf{A}_{2}} e^{\tau \mathbf{A}_{1} / 2}\right)^{m}+\mathcal{O}\left(\tau^{3}\right){ }^{30}$ where $\tau=t / m$, $\mathbf{A}=\mathbf{A}_{1}+\mathbf{A}_{2}$, and

$$
\begin{aligned}
\mathbf{A}_{1} & =\left(\begin{array}{lcc}
-1 / T_{2} & 0 & 0 \\
0 & -1 / T_{2} & 0 \\
0 & 0 & -1 / T_{1}
\end{array}\right), \\
\mathbf{A}_{2} & =\left(\begin{array}{lcc}
0 & 2 \pi \xi^{z} F_{0} & 0 \\
-2 \pi \xi^{z} F_{0} & 0 & \pi h_{p}(1+\zeta)\left(2+\xi^{x}+\xi^{y}\right) F_{R} \\
0 & -\pi h_{p}(1+\zeta)\left(2+\xi^{x}+\xi^{y}\right) F_{R} & 0
\end{array}\right) .
\end{aligned}
$$

In detail, we have

$$
\begin{aligned}
e^{\tau \mathbf{A}_{1}} & =\left(\begin{array}{lcc}
e^{-1 / T_{2}} & 0 & 0 \\
0 & e^{-1 / T_{2}} & 0 \\
0 & 0 & e^{-1 / T_{1}}
\end{array}\right), \\
e^{\tau \mathbf{A}_{2}} & =\left(\begin{array}{lcc}
1-(b / \Omega)^{2}(1-\cos \tau \Omega) & (b / \Omega) \sin \tau \Omega & \left(a b / \Omega^{2}\right)(1-\cos \tau \Omega) \\
-(b / \Omega) \sin \tau \Omega & \cos \tau \Omega & (a / \Omega) \sin \tau \Omega \\
\left(a b / \Omega^{2}\right)(1-\cos \tau \Omega) & -(a / \Omega) \sin \tau \Omega & 1-(a / \Omega)^{2}(1-\cos \tau \Omega)
\end{array}\right),
\end{aligned}
$$

where $a=2 \pi \xi^{z} F_{0}, b=\pi h_{p}(1+\zeta)\left(1+\xi^{x}+\xi^{y}\right) F_{R}$, and $\Omega=\left(a^{2}+b^{2}\right)^{1 / 2}$.

${ }^{1}$ A. Morello, P. C. E. Stamp, and I. S. Tupitsyn, Phys. Rev. Lett. 97, 207206 (2006).

${ }^{2}$ A. Leggett, S. Chakravarty, A. Dorsey, M. Fisher, A. Garg, and W. Zwerger, Rev. Mod. Phys. 59, 1 (1987).

${ }^{3}$ U. Weiss, Quantum Dissipative Systems (World Scientific, Singapore, 1999).

${ }^{4}$ N. V. Prokof'ev and P. C. E. Stamp, Rep. Prog. Phys. 63, 669 (2000). ${ }^{5}$ R. Alicki, M. Horodecki, P. Horodecki, and R. Horodecki, Phys. Rev. A 65, 062101 (2002).

${ }^{6}$ B. M. Terhal and G. Burkard, Phys. Rev. A 71, 012336 (2005).

${ }^{7}$ R. Klesse and S. Frank, Phys. Rev. Lett. 95, 230503 (2005).

${ }^{8}$ E. Novais and H. U. Baranger, Phys. Rev. Lett. 97, 040501 (2006).

${ }^{9}$ A. Abragam, The Principles of Nuclear Magnetism (Clarendon, Oxford, 1961).

${ }^{10}$ J. Villain, F. Hartman-Boutron, R. Sessoli, and A. Rettori, Europhys. Lett. 27, 159 (1994).

${ }^{11}$ A. Würger, J. Phys.: Condens. Matter 10, 10075 (1998).

${ }^{12}$ M. N. Leuenberger and D. Loss, Phys. Rev. B 61, 1286 (2000).

${ }^{13}$ N. V. Prokof'ev and P. C. E. Stamp, J. Low Temp. Phys. 104, 143 (1996).

${ }^{14}$ K. De Raedt, K. Michielsen, H. De Raedt, B. Trieu, G. Arnold, M. Richter, T. Lippert, H. Watanabe, and N. Ito, Comput. Phys. Commun. 176, 121 (2007).

${ }^{15}$ R. Boscaino, F. M. Gelardi, and J. P. Korb, Phys. Rev. B 48, 7077 (1993).

${ }^{16}$ R. N. Shakhmuratov, F. M. Gelardi, and M. Cannas, Phys. Rev. Lett. 79, 2963 (1997).
${ }^{17}$ S. Agnello, R. Boscaino, M. Cannas, F. M. Gelardi, and R. N. Shakhmuratov, Phys. Rev. A 59, 4087 (1999).

${ }^{18}$ S. Bertaina, S. Gambarelli, A. Tkachuk, N. KurkinI, B. Malkin, A. Stepanov, and B. Barbara, Nat. Nanotechnol. 2, 39 (2007).

${ }^{19}$ S. Bertaina, S. Gambarelli, T. Mitra, B. Tsukerblat, A. Muller, and B. Barbara, Nature (London) 453, 203 (2008).

${ }^{20}$ S. Bertaina, L. Chen, N. Groll, J. Van Tol, N. S. Dalal, and I. Chiorescu, Phys. Rev. Lett. 102, 050501 (2009).

${ }^{21}$ S. Bertaina, J. H. Shim, S. Gambarelli, B. Z. Malkin, and B. Barbara, Phys. Rev. Lett. 103, 226402 (2009).

${ }^{22} \mathrm{H}$. De Raedt and K. Michielsen, in Handbook of Theoretical and Computational Nanotechnology, edited by M. Rieth and W. Schommers (American Scientific, Los Angeles, 2006), pp. 2-48.

${ }^{23} \mathrm{~J}$. Weil, J. Bolton, and J. Wertz, Electron Paramagnetic Resonance: Elementary Theory and Practical Applications (Wiley, New York, 1994).

${ }^{24}$ E. I. Baibekov, Pis'ma v Zh. Eksper. Teoret. Fiz. 93, 323 (2011).

${ }^{25}$ E. Baibekov, I. Kurkin, M. Gafurov, B. Endeward, R. Rakhmatullin, and G. Mamin, J. Magn. Reson. 209, 61 (2011).

${ }^{26}$ J. A. Schreier et al., Phys. Rev. B 77, 180502 (2008).

${ }^{27}$ H. De Raedt, Comput. Phys. Rep. 7, 1 (1987).

${ }^{28}$ P. W. Anderson and P. R. Weiss, Rev. Mod. Phys. 25, 269 (1953).

${ }^{29}$ S. Takahashi, I. S. Tupitsyn, J. van Tol, C. C. Beedle, D. N. Hendrickson, and P. C. E. Stamp, Nature (London) 476, 76 (2011).

${ }^{30}$ M. Suzuki, J. Math. Phys. 26, 601 (1985). 